\title{
Estimating the Gerber-Shiu Expected Discounted Penalty Function for Lévy Risk Model
}

\author{
Yujuan Huang $\mathbb{D}^{1},{ }^{1}$ Wenguang Yu $\mathbb{D}^{1},{ }^{2}$ Yu Pan, ${ }^{3}$ and Chaoran $\mathrm{Cui}^{4}$ \\ ${ }^{1}$ School of Science, Shandong Jiaotong University, Jinan, Shandong 250357, China \\ ${ }^{2}$ School of Insurance, Shandong University of Finance and Economics, Jinan, Shandong 250014, China \\ ${ }^{3}$ College of Mathematics and Statistics, Chongqing University, Chongqing 401331, China \\ ${ }^{4}$ School of Computer Science \& Technology, Shandong University of Finance and Economics, Jinan, Shandong 250014, China
}

Correspondence should be addressed to Wenguang Yu; yuwg@sdufe.edu.cn

Received 11 December 2018; Accepted 13 February 2019; Published 2 May 2019

Academic Editor: Maria Alessandra Ragusa

Copyright (c) 2019 Yujuan Huang et al. This is an open access article distributed under the Creative Commons Attribution License, which permits unrestricted use, distribution, and reproduction in any medium, provided the original work is properly cited.

\begin{abstract}
This paper studies the statistical estimation of the Gerber-Shiu discounted penalty functions in a general spectrally negative Lévy risk model. Suppose that the claims process and the surplus process can be observed at a sequence of discrete time points. Using the observed data, the Gerber-Shiu functions are estimated by the Laguerre series expansion method. Consistent properties are studied under the large sample setting, and simulation results are also presented when the sample size is finite.
\end{abstract}

\section{Introduction}

In this paper, the cash flow of an insurance company is described by the following spectrally negative Lévy process:

$$
U_{t}=u+c t+\sigma B_{t}-X_{t}, \quad t \geq 0 .
$$

Here, $u \geq 0$ denotes the initial reserve and $c>0$ is the rate of premium. The process $\left\{B_{t}\right\}_{t \geq 0}$ is a standard Brownian motion and $\sigma>0$ is a constant. The claims process $\left\{X_{t}\right\}_{t \geq 0}$ is a Lévy subordinator, whose Laplace exponent is defined by

$$
\psi(s)=\frac{1}{t} \ln E\left[e^{-s X_{t}}\right]=\int_{0}^{\infty}\left(e^{-s x}-1\right) \nu(x) d x,
$$

$$
s \geq 0 \text {. }
$$

Here, $v$ is a Lévy density on $(0, \infty)$ satisfying the usual condition $\int_{0}^{\infty}\left(1 \wedge x^{2}\right) v(x) d x<\infty$ and the additional condition $\mu_{1}:=\int_{0}^{\infty} x v(x) d x<\infty$. Note that the condition $\mu_{1}<\infty$ ensures that the expected aggregate claims are finite over any finite time interval. Finally, let us suppose that the processes $\left\{B_{t}\right\}_{t \geq 0}$ and $\left\{X_{t}\right\}_{t \geq 0}$ are mutually independent.
In insurance risk theory, one object of interest is the ruin time $\tau$, which is defined by

$$
\tau=\inf \left\{t \geq 0: U_{t} \leq 0\right\} .
$$

Set $\tau=\infty$ if $U_{t}>0$ for all $t \geq 0$. In order to avoid ruin is a deterministic event, it is assumed that the following net profit condition holds true throughout this paper.

Condition 1. The premium rate $c>\mu_{1}$.

In this paper, we use the Gerber-Shiu expected discounted penalty function to discuss the ruin problems. This function is defined by

$$
\phi(u)=E\left[e^{-\delta \tau} w\left(\left|U_{\tau}\right|\right) \mathbf{1}_{(\tau<\infty)} \mid U_{0}=u\right], \quad u \geq 0,
$$

where $\delta \geq 0$ is a constant interest force, $w$ is a nonnegative measurable penalty function of the deficit at ruin, and $\mathbf{1}_{A}$ is an indicator function of an event $A$. Note that ruin can be caused by oscillation or claims. Then we can decompose the Gerber-Shiu function as follows:

$$
\phi(u)=w(0) \phi_{d}(u)+\phi_{c}(u),
$$


where

$$
\phi_{d}(u)=E\left[e^{-\delta \tau} \mathbf{1}_{\left(\tau<\infty, U_{\tau}=0\right)} \mid U_{0}=u\right]
$$

is the Laplace transform of ruin time when ruin is caused by oscillation and

$$
\phi_{c}(u)=E\left[e^{-\delta \tau} w\left(\left|U_{\tau}\right|\right) \mathbf{1}_{\left(\tau<\infty, U_{\tau}<0\right)} \mid U_{0}=u\right]
$$

is the expected discounted penalty function when ruin is due to claims.

In insurance risk theory, the Gerber-Shiu function introduced by Gerber and Shiu [1] is a powerful tool for solving ruin problems. It should be emphasized that most of the existing literatures mainly pursue explicit formulae of Gerber-Shiu functions under various models. For example, Gerber and Shiu [1] studied the classical risk model; Li and Garrido $[2,3]$ discussed the Sparre Andersen risk models. In risk theory, the Lévy process is often used to model the surplus process of an insurance company, and a large number of results to Gerber-Shiu function have been made by researchers. Garrido and Morales [4] used Laplace transform to investigate the classical Gerber-Shiu function. Biffis and Morales [5] generalized the Gerber-Shiu function to pathdependent penalties. Chau et al. [6] used the Fourier-cosine method to evaluate the Gerber-Shiu function. For more studies on Gerber-Shiu function, the interested readers are referred to Yin and Wang [7, 8], Asmussen and Albrecher [9], Chi [10], Wang et al. [11], Chi and Lin [12], Zhao and Yin $[13,14]$, Shen et al. [15], Yu [16-18], Yin and Yuen [19, 20], Zhao and Yao [21], Zheng et al. [22], Huang et al. [23], Li et al. [24], Zhang et al. [25], Yu et al. [26], Zeng et al. [27, 28], Li et al. [29], and Dong et al. [30].

Contributing to Gerber-Shiu function mentioned above, it is commonly assumed that the probabilistic law of the surplus process is known, so that some analytic formulae can be derived. However, from the practical point of view, the insurance company does not know the probabilistic law, instead the surplus data and claim sizes data are often known. Hence, it is of importance to develop some methods for estimating the Gerber-Shiu type risk measures from the past data.

Over the past decade, the importance of statistical estimation of Gerber-Shiu function has advanced rapidly. As a special type of Gerber-Shiu functions, the ruin probability is estimated by Mnatsakanov et al. [31], Masiello [32], and Zhang et al. [33] under the classical compound Poisson risk model. Further, Zhang [34] and Yang et al. [35] estimated the finite ruin probability by double Fourier transform. For the general Gerber-Shiu function, Shimizu [36] estimated it by Laplace inversion in the compound Poisson insurance risk model. Zhang $[37,38]$ proposed an estimator by Fourier-sinc and Fourier-cosine series expansion. Zhang and Su [39, 40] and $\mathrm{Su}$ et al. [41] proposed a more efficient estimator by Laguerre series expansion method. In addition, the estimation of ruin probability and Gerber-Shiu function in the Lévy risk model has also attracted the attention of scholars. For example, Shimizu [42] estimated the Gerber-Shiu function in a general spectrally negative Lévy process. Zhang and Yang $[43,44]$ estimated the ruin probability in a pure jump Lévy risk model. Shimizu and Zhang [45] estimated the GerberShiu function in the pure jump Lévy risk model. Moreover, the study is a useful tool not only for the estimation of ruin probability by invasive exotic species on habitat and natural systems; see Wang and Yin [46], Yuen and Yin [47], Dong and Yin [48], Yin et al. [49], Wang et al. [50], Zhao et al. [51], Pavone et al. [52], Zhou et al. [53], Costa and Pavone [54, 55], and Yin [56], but also for explaining the dynamics of the disappearance of the plants of the past and changing of biodiversity and wavelet analysis, see Costa et al. [57], Pulvirenti et al. [58, 59], Lemarié and Meyer [60], and Daubechies [61].

The remainder of this paper is organized as follows. In Section 2, we provide some known results on Gerber-Shiu function, which are useful for constructing the estimator. In Section 3, we give an estimator by the Laguerre series expansion method, where both the surplus data and the aggregate claims data are used. Some consistent properties of the estimator are investigated in Section 4. Finally, in Section 5, we provide numerical examples to illustrate the efficiency of the method.

\section{Preliminaries on Gerber-Shiu Functions}

2.1. The Laguerre Basis. In this paper, we use $L^{1}\left(\mathbb{R}_{+}\right)$and $L^{2}\left(\mathbb{R}_{+}\right)$to denote the spaces of absolutely integrable functions and square integrable functions on the positive half line, respectively. For any $p, q \in L^{2}\left(\mathbb{R}_{+}\right)$, the inner product and the $L^{2}$-norm are defined by

$$
\langle p, q\rangle=\int_{0}^{\infty} p(x) q(x) d x, \quad\|p\|=\sqrt{\langle p, p\rangle .}
$$

For the Laguerre functions, they are defined by

$$
\varphi_{k}(x)=\sqrt{2} L_{k}(2 x) e^{-x}, \quad x \geq 0 ; k=0,1, \ldots,
$$

where $\left\{L_{k}\right\}$ are the Laguerre polynomials given by

$$
L_{k}(x)=\sum_{j=0}^{k}(-1)^{j}\left(\begin{array}{l}
k \\
j
\end{array}\right) \frac{x^{j}}{j !}, \quad x \geq 0 .
$$

It is known that $\left\{\varphi_{k}\right\}_{k \geq 0}$ is an orthonormal basis of $L^{2}\left(\mathbb{R}_{+}\right)$ such that

$$
\begin{aligned}
\left\|\varphi_{k}\right\| & =1 ; \\
\left\langle\varphi_{k}, \varphi_{j}\right\rangle & =0
\end{aligned}
$$

for $k \neq j$.

Hence, each $f \in L^{2}\left(\mathbb{R}_{+}\right)$can be developed on the Laguerre basis, i.e.,

$$
f(x)=\sum_{k=0}^{\infty} a_{f, k} \varphi_{k}(x), \quad x \geq 0,
$$

where $a_{f, k}=\left\langle f, \varphi_{k}\right\rangle, k=0,1, \ldots$

The following properties of Laguerre functions are useful, which can be found in Abrumowitz and Stegun [62]. 
(1) $\sup _{x \geq 0}\left|\varphi_{k}(x)\right| \leq \sqrt{2}, \forall k \geq 0$.

(2) $\int_{0}^{x} \varphi_{k}(x-y) \varphi_{j}(y) d y=(1 / \sqrt{2})\left[\varphi_{k+j}(x)-\varphi_{k+j+1}(x)\right]$.

2.2. Review on Gerber-Shiu Function. In this subsection, we present some necessary results on the Gerber-Shiu function, which are useful for constructing the estimator. First, we consider the root of the following equation (in s):

$$
c s+\frac{\sigma^{2}}{2} s^{2}+\psi(s)=\delta .
$$

It is known that (13) has a positive root, say $\rho$, and we have $\rho=0$ as $\delta=0$. Set $\theta=2 c / \sigma^{2}+\rho$.

By Corollary 4.1 in Biffis and Morales [5] we have

$$
\phi(u)=\left[h_{c}(u)+w(0) h_{d}(u)\right] * \sum_{n=0}^{\infty} g^{* n}(u), \quad u \geq 0,
$$

where $*$ denotes the convolution operator, $h_{d}(u)=e^{-\theta u}$, and

$$
\begin{gathered}
g(x)=\frac{2}{\sigma^{2}} \int_{0}^{x} e^{-\theta(x-y)} \int_{y}^{\infty} e^{-\rho(z-y)} \nu(z) d z d y, \\
h_{c}(u)=\frac{2}{\sigma^{2}} \int_{0}^{u} e^{-\theta(u-y)} \int_{y}^{\infty} e^{-\rho(z-y)} \varsigma(z) d z d y,
\end{gathered}
$$

where $\varsigma(z)=\int_{z}^{\infty} w(x-z) v(x) d x, z \geq 0$. Furthermore, from the deduction in Biffis and Morales [5] one easily obtains

$$
\phi_{d}(u)=h_{d}(u) * \sum_{n=0}^{\infty} g^{* n}(u)
$$

and

$$
\phi_{c}(u)=h_{c}(u) * \sum_{n=0}^{\infty} g^{* n}(u) .
$$

It follows from (17) that

$$
\begin{aligned}
\phi_{d}(u) & =h_{d}(u)+\left(h_{d}(u) * \sum_{n=1}^{\infty} g^{*(n-1)}(u)\right) * g(u) \\
& =h_{d}(u)+g * \phi_{d}(u),
\end{aligned}
$$

i.e., $\phi_{d}$ satisfies the following renewal equation:

$$
\phi_{d}(u)=\int_{0}^{u} \phi_{d}(u-x) g(x) d x+h_{d}(u), \quad u \geq 0 .
$$

Similarly, we can obtain

$$
\phi_{c}(u)=\int_{0}^{u} \phi_{c}(u-x) g(x) d x+h_{c}(u), \quad u \geq 0 .
$$

It should be emphasized that (20) and (21) are both defective renewal equations, since $0<\int_{0}^{\infty} g(x) d x<1$ under Condition 1.

For purpose of employing the Laguerre series expansion, we suppose the following condition.
Condition 2. The functions $\phi_{d}, \phi_{c}, g, h_{d}, h_{c}$ are all square integrable.

Under Condition 2, we have the following Laguerre series expansion formulae:

$$
\begin{aligned}
& \phi_{d}(u)=\sum_{k=0}^{\infty} a_{\phi_{d}, k} \varphi_{k}(u), \\
& \phi_{c}(u)=\sum_{k=0}^{\infty} a_{\phi_{c}, k} \varphi_{k}(u),
\end{aligned}
$$

and

$$
\begin{aligned}
& g(x)=\sum_{k=0}^{\infty} a_{g, k} \varphi_{k}(x), \\
& h_{d}(u)=\sum_{k=0}^{\infty} a_{h_{d}, k} \varphi_{k}(u), \\
& h_{c}(u)=\sum_{k=0}^{\infty} a_{h_{c}, k} \varphi_{k}(u) .
\end{aligned}
$$

Substituting the above five expressions into (20) and (21) and using a similar method as in Zhang and Su [39], we can obtain for $k=0,1,2, \ldots$,

$$
a_{\phi_{d}, k}=\sum_{j=0}^{k} 2^{-1 / 2}\left[a_{g, k-j}-a_{g, k-j-1}\right] a_{\phi_{d}, j}+a_{h_{d}, k}
$$

and

$$
a_{\phi_{c}, k}=\sum_{j=0}^{k} 2^{-1 / 2}\left[a_{g, k-j}-a_{g, k-j-1}\right] a_{\phi_{c}, j}+a_{h_{c}, k} .
$$

The above linear systems can further be rewritten in matrix form as follows:

$$
\begin{aligned}
\boldsymbol{A}_{\infty} \overrightarrow{\boldsymbol{a}}_{\phi_{d}, \infty} & =\overrightarrow{\boldsymbol{a}}_{h_{d}, \infty}, \\
\boldsymbol{A}_{\infty} \overrightarrow{\boldsymbol{\phi}}_{\phi_{c}, \infty} & =\overrightarrow{\boldsymbol{a}}_{h_{c}, \infty},
\end{aligned}
$$

where $\overrightarrow{\boldsymbol{a}}_{\phi_{d}, \infty}=\left(a_{\phi_{d}, 0}, a_{\phi_{d}, 1}, \ldots\right)^{T}, \overrightarrow{\boldsymbol{a}}_{\phi_{c}, \infty}=\left(a_{\phi_{c}, 0}, a_{\phi_{c}, 1}, \ldots\right)^{T}$, $\overrightarrow{\boldsymbol{a}}_{h_{d}, \infty}=\left(a_{h_{d}, 0}, a_{h_{d}, 1}, \ldots\right)^{T}, \overrightarrow{\boldsymbol{a}}_{h_{c}, \infty}=\left(a_{h_{c}, 0}, a_{h_{c}, 1}, \ldots\right)^{T}$, and the elements in $\boldsymbol{A}_{\infty}$ are given by

$$
\left[\boldsymbol{A}_{\infty}\right]_{k, j}= \begin{cases}1-2^{-1 / 2} a_{g, 0}, & \text { if } k=j, \\ 2^{-1 / 2}\left[a_{g, k-j-1}-a_{g, k-j}\right], & \text { if } k>j, \\ 0, & \text { otherwise. }\end{cases}
$$

Furthermore, put $\overrightarrow{\boldsymbol{a}}_{\phi_{d}, m}=\left(a_{\phi_{d}, 0}, \ldots, a_{\phi_{d}, m}\right)^{T}, \overrightarrow{\boldsymbol{a}}_{\phi_{c}, m}=\left(a_{\phi_{c}, 0}\right.$, $\left.\ldots, a_{\phi_{c}, m}\right)^{T}, \overrightarrow{\boldsymbol{a}}_{h_{d}, m}=\left(a_{h_{d}, 0}, \ldots, a_{h_{d}, m}\right)^{T}, \overrightarrow{\boldsymbol{a}}_{h_{c}, m}=\left(a_{h_{c}, 0}, a_{h_{c}, 1}\right.$, $\left.\ldots, a_{h_{c}, m}\right)^{T}$, and $\boldsymbol{A}_{m}=\left(\left[\boldsymbol{A}_{\infty}\right]_{i, j}\right)_{0 \leq i, j \leq m}$. Then we can truncate the matrix and vectors in (26) to obtain

$$
\begin{aligned}
\boldsymbol{A}_{m} \overrightarrow{\boldsymbol{a}}_{\phi_{d}, m} & =\overrightarrow{\boldsymbol{a}}_{h_{d}, m}, \\
\boldsymbol{A}_{m} \overrightarrow{\boldsymbol{a}}_{\phi_{c}, m} & =\overrightarrow{\boldsymbol{a}}_{h_{c}, m} .
\end{aligned}
$$


Note that the matrix $\boldsymbol{A}_{m}$ is a lower triangular Toeplitz matrix, and all the diagonal elements are positive since $1-2^{-1 / 2} a_{g, 0}>$ $1-\int_{0}^{\infty} g(x) d x>0$. Hence, $\boldsymbol{A}_{m}$ is nonsingular and explicitly invertible. Solving equations in (28) we get

$$
\begin{aligned}
& \overrightarrow{\boldsymbol{a}}_{\phi_{d}, m}=\boldsymbol{A}_{m}{ }^{-1} \overrightarrow{\boldsymbol{a}}_{h_{d}, m}, \\
& \overrightarrow{\boldsymbol{a}}_{\phi_{c}, m}=\boldsymbol{A}_{m}{ }^{-1} \overrightarrow{\boldsymbol{a}}_{h_{c}, m} .
\end{aligned}
$$

Finally, we can approximate the Gerber-Shiu function by

$$
\begin{aligned}
& \phi_{d}(u) \approx \phi_{d, m}(u):=\sum_{k=0}^{m} a_{\phi_{d}, k} \varphi_{k}(u), \\
& \phi_{c}(u) \approx \phi_{c, m}(u):=\sum_{k=0}^{m} a_{\phi_{c}, k} \varphi_{k}(u) .
\end{aligned}
$$

\section{Estimating the Gerber-Shiu Function}

Suppose that we can observe the surplus process $\left\{U_{t}\right\}$ and the claims process $\left\{X_{t}\right\}$ at a sequence of discrete time points, so that the following samples are available:

$$
\begin{aligned}
& U^{n}:=\left\{U_{k \Delta}: k=1,2, \ldots, n\right\}, \\
& \boldsymbol{X}^{n}:=\left\{X_{k \Delta}: k=1,2, \ldots, n\right\},
\end{aligned}
$$

where $\Delta:=\Delta_{n}>0$ is a sampling step. Further note that the initial values $U_{0}=u$ and $X_{0}=0$ and the premium rate are known, but the diffusion volatility parameter $\sigma^{2}$ and Lévy density $\nu$ are both known. It is convenient to use the following samples:

$$
\begin{aligned}
Z^{n} & :=\left\{Z_{k \Delta}=X_{k \Delta}-X_{(k-1) \Delta}: k=1,2, \ldots, n\right\}, \\
W^{n} & :=\left\{W_{k \Delta}=U_{k \Delta}-U_{(k-1) \Delta}-c \Delta\right. \\
& \left.+\left(X_{k \Delta}-X_{(k-1) \Delta}\right): k=1,2, \ldots, n\right\} .
\end{aligned}
$$

In this paper, the observation is based on high frequency in a long term, which means that the following condition holds true.

Condition 3. The sampling interval satisfies

$$
\begin{gathered}
\lim _{n \rightarrow \infty} \Delta=0, \\
\lim _{n \longrightarrow \infty} n \Delta=\infty .
\end{gathered}
$$

We shall use the notation $\mu_{k}:=\int_{0}^{\infty} x^{k} \nu(x) d x, k \geq 1$, provided that such moment is finite. That is to say, we shall use the following condition.

Condition $4(k) . \mu_{k}:=\int_{0}^{\infty} x^{k} \nu(x) d x<\infty, k=1,2, \ldots$

Note that Condition $4(l)$ implies Condition $4(k)$ if $l \geq$ $k, k, l=1,2, \ldots$ By Proposition 2.2 in Comte and GenonCatalot [63] we have

$$
E Z_{1}^{k}=\mu_{k} \Delta+o(\Delta)
$$

under Conditions 3 and $4(k)$.
The following two conditions are also useful for studying the consistent properties.

Condition 5. The Lévy density is continuous on $(0, \infty)$, and for some $\alpha \in(0,1)$,

$$
\lim _{\Delta \longrightarrow 0} \Delta^{2-\alpha} \nu(\Delta)=0 .
$$

Condition 6. For some $\kappa, \kappa^{\prime}>0$,

$$
\begin{gathered}
w(x) \leq C(1+x)^{\kappa}, \\
w^{\prime}(x) \leq C(1+x)^{\kappa^{\prime}} .
\end{gathered}
$$

Now we study how to estimate the Gerber-Shiu function. First, we estimate $\sigma^{2}$ and $\nu$. For $\sigma^{2}$, we can estimate it by

$$
\widehat{\sigma}^{2}=\frac{1}{n \Delta} \sum_{k=1}^{n} W_{k \Delta}^{2}
$$

which is an unbiased estimator since

$$
E \widehat{\sigma}^{2}=\frac{1}{\Delta} E\left[W_{\Delta}^{2}\right]=\frac{1}{\Delta} E\left[\sigma^{2} B_{\Delta}^{2}\right]=\sigma^{2} .
$$

Furthermore, it is easily seen that

$$
\widehat{\sigma}^{2}-\sigma^{2}=O_{p}\left((n \Delta)^{-1 / 2}\right) .
$$

For the Lévy density $v$, it is known that under Conditions 3 we have $(1 / n \Delta) \sum_{k=1}^{n} \delta_{Z_{k}}(d x)$ converges weakly to the measure $v(x) d x$, where $\delta_{x}$ denotes the Dirac measure at $x$. Using this result and noting that $\psi(s)=\int_{0}^{\infty}\left(e^{-s x}-1\right) \nu(x) d x$, we can estimate the Laplace exponent by

$$
\widehat{\psi}(s)=\frac{1}{n \Delta} \sum_{k=1}^{n}\left[e^{-s Z_{k}}-1\right] .
$$

Recalling that $\rho$ is the positive root of equation, then the estimator of $\rho$, say $\hat{\rho}$, is defined to be the positive root of the following estimating equation:

$$
c s+\frac{\widehat{\sigma}^{2}}{2} s^{2}+\widehat{\psi}(s)=\delta .
$$

We set $\widehat{\rho}=0$ as $\delta=0$.

Lemma 7. Suppose that Conditions 1, 2, and 4(2) hold true. Then for $\delta>0$ we have

$$
\widehat{\rho}-\rho=O_{p}\left((n \Delta)^{-1 / 2}+\Delta\right) .
$$

Proof. This can be proved using the same arguments as in Shimizu [42].

Let us consider how to estimate $a_{h_{d}, k}, a_{h_{c}, k}$, and $a_{g, k}$. First, for $a_{h_{d}, k}$ we have

$$
a_{h_{d}, k}=\int_{0}^{\infty} e^{-\theta u} \varphi_{k}(u) d u=\sqrt{2} \frac{(\theta-1)^{k}}{(1+\theta)^{k+1}},
$$


which yields the following estimator:

$$
\widehat{a}_{h_{d}, k}=\sqrt{2} \frac{(\widehat{\theta}-1)^{k}}{(1+\hat{\theta})^{k+1}},
$$

where $\widehat{\theta}=2 c / \widehat{\sigma}^{2}+\widehat{\rho}$. Next, by changing the order of integrals we have

$$
\begin{aligned}
& a_{h_{c}, k}=\int_{0}^{\infty} h_{c}(u) \varphi_{k}(u) d u=\frac{2}{\sigma^{2}} \\
& \cdot \int_{0}^{\infty} \int_{0}^{u} e^{-\theta(u-y)} \int_{y}^{\infty} e^{-\rho(z-y)} w(z) d z d y \varphi_{k}(u) d u \\
& :=\frac{2}{\sigma^{2}} \int_{0}^{\infty} R_{k}(x, \rho, \theta) \nu(x) d x,
\end{aligned}
$$

where

$$
\begin{gathered}
R_{k}(x, \rho, \theta)=\int_{0}^{x} \int_{0}^{z} \int_{y}^{\infty} e^{-\theta(u-y)} e^{-\rho(z-y)} \varphi_{k}(u) \\
\cdot w(x-z) d u d y d z .
\end{gathered}
$$

Then we can estimate $a_{h_{c}, k}$ by

$$
\widehat{a}_{h_{c}, k}=\frac{2}{\widehat{\sigma}^{2}} \frac{1}{n \Delta} \sum_{j=1}^{n} R_{k}\left(Z_{j}, \widehat{\rho}, \widehat{\theta}\right) .
$$

Finally, for $a_{g, k}$ we have

$$
\begin{aligned}
a_{g, k} & =\int_{0}^{\infty} g(x) \varphi_{k}(x) d x=\frac{2}{\sigma^{2}} \\
\cdot & \int_{0}^{\infty} \int_{0}^{x} e^{-\theta(x-y)} \int_{y}^{\infty} e^{-\rho(x-y)} \nu(z) d z d y \varphi_{k}(x) d x \\
& :=\frac{2}{\sigma^{2}} \int_{0}^{\infty} Q_{k}(z, \rho, \theta) \nu(z) d z,
\end{aligned}
$$

where

$$
Q_{k}(z, \rho, \theta)=\int_{0}^{z} \int_{y}^{\infty} e^{-\theta(x-y)} e^{-\rho(z-y)} \varphi_{k}(x) d x d y .
$$

Then we can estimate $a_{g, k}$ by

$$
\widehat{a}_{g, k}=\frac{2}{\widehat{\sigma}^{2}} \frac{1}{n \Delta} \sum_{j=1}^{n} Q_{k}\left(Z_{j}, \widehat{\rho}, \widehat{\theta}\right) .
$$

Note that $Q_{k}(z, \rho, \theta)$ can be explicitly calculated as follows:

$$
\begin{aligned}
& Q_{k}(z, \rho, \theta)=\sqrt{2} \sum_{j=0}^{k}(-2)^{j} \\
& \cdot\left(\begin{array}{c}
k \\
j
\end{array}\right) \int_{0}^{z} \int_{y}^{\infty} e^{-\theta(x-y)} e^{-\rho(z-y)} \frac{x^{j}}{j !} e^{-x} d x d y \\
& =\sqrt{2} \sum_{j=0}^{k}(-2)^{j}\left(\begin{array}{c}
k \\
j
\end{array}\right) e^{-\rho z} \sum_{i=0}^{j} \frac{1}{(1+\theta)^{j+1-i}}\left\{\frac{1}{(1-\rho)^{i+1}}\right. \\
& \left.-\sum_{l=0}^{i} \frac{1}{(1-\rho)^{i+1-l}} \frac{z^{l}}{l !} e^{-(1-\rho) z}\right\} .
\end{aligned}
$$

As for the function $R_{k}(x, \rho, \theta)$, it can also be explicitly calculated for some special cases of penalty function. For example, if $w \equiv 1$, we have

$$
\begin{array}{r}
R_{k}(x, \rho, \theta)=\sqrt{2} \sum_{j=0}^{k} \sum_{i=0}^{j}\left(\begin{array}{l}
k \\
j
\end{array}\right) \frac{(-2)^{j}}{(1+\theta)^{j+1-i}} \frac{1}{(1-\rho)^{i+1}} \\
\cdot\left\{\frac{1}{\rho}\left(1-e^{-\rho x}\right)-\sum_{l=0}^{i}(1-\rho)^{l}\left(1-\sum_{r=0}^{l} \frac{x^{r}}{r !} e^{-x}\right)\right\} .
\end{array}
$$

For the vectors $\overrightarrow{\boldsymbol{a}}_{h_{d}, m}, \overrightarrow{\boldsymbol{a}}_{h_{c}, m}$, and $\overrightarrow{\boldsymbol{a}}_{g, m}$, we estimate them by $\widehat{\overrightarrow{\boldsymbol{a}}}_{h_{d}, m}=\left(\widehat{a}_{h_{d}, 0}, \widehat{a}_{h_{d}, 1}, \ldots, \widehat{a}_{h_{d}, m}\right)^{T}, \widehat{\overrightarrow{\boldsymbol{a}}}_{h_{c}, m}=\left(\widehat{a}_{h_{c}, 0}, \widehat{a}_{h_{c}, 1}, \ldots\right.$, $\left.\widehat{a}_{h_{c}, m}\right)^{T}$, and $\widehat{\overrightarrow{\boldsymbol{a}}}_{g, m}=\left(\widehat{a}_{g, 0}, \widehat{a}_{g, 1}, \ldots, \widehat{a}_{g, m}\right)^{T}$, respectively. As for the matrix $\boldsymbol{A}_{m}$, we estimate it by $\widehat{\boldsymbol{A}}_{m}$, where the elements $a_{g, k}$ in $A_{m}$ are replaced by $\widehat{a}_{g, k}$. Finally, we estimate the GerberShiu functions by

$$
\begin{gathered}
\widehat{\phi}_{d, m}(u)=\sum_{k=0}^{m} \widehat{a}_{\phi_{d, k}} \varphi_{k}(u), \\
\widehat{\phi}_{c, m}(u)=\sum_{k=0}^{m} \widehat{a}_{\phi_{c, k}} \varphi_{k}(u),
\end{gathered}
$$

where

$$
\begin{aligned}
& \widehat{\overrightarrow{\boldsymbol{a}}}_{\phi_{d, m}}:=\left(\widehat{a}_{\phi_{d, 0}}, \widehat{a}_{\phi_{d, 1}}, \ldots, \widehat{a}_{\phi_{d, m}}\right)=\widehat{\boldsymbol{A}}_{m}^{-1} \widehat{\overrightarrow{\boldsymbol{a}}}_{h_{d, m}}, \\
& \widehat{\overrightarrow{\boldsymbol{a}}}_{\phi_{c, m}}:=\left(\widehat{a}_{\phi_{c, 0}}, \widehat{a}_{\phi_{c, 1}}, \ldots, \widehat{a}_{\phi_{c, m}}\right)=\widehat{\boldsymbol{A}}_{m}^{-1} \widehat{\widehat{\boldsymbol{a}}}_{h_{c, m}} .
\end{aligned}
$$

\section{Consistent Properties}

In this section, we investigate the asymptotic properties of the proposed estimators as $m, n \longrightarrow \infty$. In the sequel, we denote by $C$ a generic positive constant that may vary at different steps. Our goal is to study the errors of $\left\|\hat{\phi}_{d, m}-\phi_{d}\right\|$ and $\left\|\widehat{\phi}_{c, m}-\phi_{c}\right\|$. By Pythagoras principle, we have

$$
\left\|\widehat{\phi}_{d, m}-\phi_{d}\right\|^{2}=\left\|\hat{\phi}_{d, m}-\phi_{d, m}\right\|^{2}+\left\|\phi_{d, m}-\phi_{d}\right\|^{2}
$$

and

$$
\left\|\widehat{\phi}_{c, m}-\phi_{c}\right\|^{2}=\left\|\widehat{\phi}_{c, m}-\phi_{c, m}\right\|^{2}+\left\|\phi_{c, m}-\phi_{c}\right\|^{2} .
$$

Note that $\left\|\widehat{\phi}_{d, m}-\phi_{d, m}\right\|$ and $\left\|\widehat{\phi}_{c, m}-\phi_{c, m}\right\|$ are statistical errors due to estimating the Laguerre coefficients and $\left\|\phi_{d, m}-\phi_{d}\right\|$ and $\left\|\phi_{c, m}-\phi_{c}\right\|$ are biases due to series truncation.

First, we consider the biases. To this end, we introduce the Sobolev-Laguerre space (Bongioanni and Torrea [64]), which is defined by

$$
\begin{aligned}
W & \left(\mathbb{R}_{+}, r, B\right) \\
& =\left\{f: \mathbb{R}_{+} \longrightarrow \mathbb{R}, f \in L^{2}\left(\mathbb{R}_{+}\right), \sum_{k=0}^{\infty} k^{r} a_{f, k}^{2} \leq B\right\},
\end{aligned}
$$


where $0<r, B<\infty$. It follows from Zhang and $\mathrm{Su}$ [39] that, under conditions $\phi_{d}, \phi_{c} \in W\left(\mathbb{R}_{+}, r, \mathscr{B}\right)$, we have

$$
\begin{aligned}
\left\|\phi_{d, m}-\phi_{d}\right\|^{2} & =O\left(m^{-r}\right), \\
\left\|\phi_{c, m}-\phi_{c}\right\|^{2} & =O\left(m^{-r}\right) .
\end{aligned}
$$

Next, we discuss the statistical errors. For a vector $\overrightarrow{\boldsymbol{b}}=$ $\left(b_{1}, \ldots, b_{n}\right)^{T}$, we denote its 2-norm by $\|\overrightarrow{\boldsymbol{b}}\|_{2}=\sqrt{\sum_{i=1}^{n}\left|b_{i}\right|^{2}}$. For a square matrix $\boldsymbol{B}=\left(b_{i j}\right)_{1 \leq i, j \leq n}$, its spectral norm is defined by $\|\boldsymbol{B}\|_{2}=\sqrt{\lambda_{\max }\left(\boldsymbol{B}^{T} \boldsymbol{B}\right)}$, where $\lambda_{\max }\left(\boldsymbol{B}^{T} \boldsymbol{B}\right)$ is the largest eigenvalue of $\boldsymbol{B}^{T} \boldsymbol{B}$. Furthermore, we denote by

$$
\|\boldsymbol{B}\|_{F}=\sqrt{\operatorname{tr}\left(\boldsymbol{B}^{T} \boldsymbol{B}\right)}=\sqrt{\sum_{i=1}^{n} \sum_{j=1}^{n} b_{i j}^{2}}
$$

the Frobenius norm. Obviously, we have $\|\boldsymbol{B}\|_{2} \leq\|\boldsymbol{B}\|_{F}$, and for any two square matrices $\boldsymbol{B}_{1}$ and $\boldsymbol{B}_{2}$ with the same dimension, $\left\|\boldsymbol{B}_{1} \boldsymbol{B}_{2}\right\|_{2} \leq\left\|\boldsymbol{B}_{1}\right\|_{2} \cdot\left\|\boldsymbol{B}_{2}\right\|_{2}$.

Now using the same arguments as in deriving (61) in Zhang and Su [39], we have

$$
\begin{aligned}
\left\|\widehat{\phi}_{d, m}-\phi_{d, m}\right\|^{2} \leq & 3\left\|A_{m}^{-1}\right\|_{2}^{2} \cdot\left\|\overrightarrow{\boldsymbol{a}}_{h_{d, m}}-\widehat{\overrightarrow{\boldsymbol{a}}}_{h_{d, m}}\right\|_{2}^{2} \\
& +3\left\|\boldsymbol{A}_{m}^{-1}-\widehat{\boldsymbol{A}}_{m}^{-1}\right\|_{2}^{2} \cdot\left\|\overrightarrow{\boldsymbol{a}}_{h_{d, m}}-\widehat{\overrightarrow{\boldsymbol{a}}}_{h_{d, m}}\right\|_{2}^{2} \\
& +3\left\|\boldsymbol{A}_{m}^{-1}-\widehat{\boldsymbol{A}}_{m}^{-1}\right\|_{2}^{2} \cdot\left\|\overrightarrow{\boldsymbol{a}}_{h_{d, m}}\right\|_{2}^{2}
\end{aligned}
$$

and

$$
\begin{aligned}
\left\|\widehat{\phi}_{c, m}-\phi_{c, m}\right\|^{2} \leq & 3\left\|\boldsymbol{A}_{m}^{-1}\right\|_{2}^{2} \cdot\left\|\overrightarrow{\boldsymbol{a}}_{h_{c, m}}-\widehat{\overrightarrow{\boldsymbol{a}}}_{h_{c, m}}\right\|_{2}^{2} \\
& +3\left\|\boldsymbol{A}_{m}^{-1}-\widehat{\boldsymbol{A}}_{m}^{-1}\right\|_{2}^{2} \cdot\left\|\overrightarrow{\boldsymbol{a}}_{h_{c, m}}-\widehat{\overrightarrow{\boldsymbol{a}}}_{h_{c, m}}\right\|_{2}^{2} \\
& +3\left\|\boldsymbol{A}_{m}^{-1}-\widehat{\boldsymbol{A}}_{m}^{-1}\right\|_{2}^{2} \cdot\left\|\overrightarrow{\boldsymbol{a}}_{h_{c, m}}\right\|_{2}^{2} .
\end{aligned}
$$

To derive upper bounds for the right hand sides of (60) and (61), we still need some lemmas.

Lemma 8. Suppose that Condition 1 holds true. Then for all $m \geq 1$, we have

$$
\left\|A_{m}^{-1}\right\|_{2} \leq \frac{c+\left(\sigma^{2} / 2\right) \rho}{c+\left(\sigma^{2} / 2\right) \rho-\mu_{1}} .
$$

Proof. Note that the infinite dimensional lower triangular Toeplitz matrix $\boldsymbol{A}$ is generated by the sequence $\left\{b_{k}\right\}_{k \geq 0}$, where

$$
\begin{aligned}
& b_{0}=1-\frac{1}{\sqrt{2}} a_{g, 0}, \\
& b_{k}=\frac{1}{\sqrt{2}}\left(a_{g, k-1}-a_{g, k}\right), \quad k=1,2, \ldots
\end{aligned}
$$

Furthermore, by Lemma C.1 in Comte et al. [65] we know that $\left\{b_{k}\right\}_{k \geq 0}$ are Fourier coefficients of the function

$$
b\left(e^{i s}\right)=\sum_{k=0}^{\infty} b_{k} e^{i s k}=1-\hat{g}\left(\frac{1+e^{i s}}{1-e^{i s}}\right), \quad s \in \mathbb{R} .
$$

For the function $b$, we have

$$
\begin{aligned}
& \inf _{z \in C}|b(z)|=\inf _{z \in \mathscr{C}}\left|1-\widehat{g}\left(\frac{1+z}{1-z}\right)\right| \\
& \geq 1-\sup _{z \in \mathscr{C}}\left|\widehat{g}\left(\frac{1+z}{1-z}\right)\right| \geq 1-\int_{0}^{\infty} g(x) d x \\
& =1-\frac{2}{\sigma^{2}} \int_{0}^{\infty} \int_{0}^{x} e^{-\theta(x-y)} \int_{y}^{\infty} e^{-\rho(z-y)} v(z) d z d y d x \\
& \geq 1-\frac{2}{\sigma^{2}} \int_{0}^{\infty} \int_{0}^{x} e^{-\theta(x-y)} \int_{y}^{\infty} v(z) d z d y d x \\
& =1-\frac{2}{\sigma^{2}}\left(\int_{0}^{\infty} e^{-\theta x} d x\right)\left(\int_{0}^{\infty} \int_{x}^{\infty} \nu(z) d z d x\right) \\
& =1-\frac{2}{\sigma^{2}} \frac{1}{\theta} \cdot \mu_{1}=1-\frac{\mu_{1}}{c+\left(\sigma^{2} / 2\right) \rho}>0,
\end{aligned}
$$

where $\mathscr{C}=\{z \in \mathbb{C}:|z|=1\}$ is the complex unit circle. Finally, by Lemma A.1 in Zhang and Su [39] we obtain

$$
\begin{aligned}
\left\|\boldsymbol{A}_{m}^{-1}\right\|_{2} & \leq \frac{2}{1-\mu_{1} /\left(c+\left(\sigma^{2} / 2\right) \rho\right)} \\
& =\frac{c+\left(\sigma^{2} / 2\right) \rho}{c+\left(\sigma^{2} / 2\right) \rho-\mu_{1}} .
\end{aligned}
$$

This completes the proof.

Lemma 9. Under Condition 2, for each $m \geq 1$ we have

$$
\begin{aligned}
\left\|\overrightarrow{\boldsymbol{a}}_{h_{d}, m}\right\|_{2}^{2} & \leq\left\|h_{d}\right\|^{2}<\infty, \\
\left\|\overrightarrow{\boldsymbol{a}}_{h_{c}, m}\right\|^{2} & \leq\left\|h_{c}\right\|^{2}<\infty .
\end{aligned}
$$

Proof. The above results hold true since

$$
\left\|\overrightarrow{\boldsymbol{a}}_{h_{d}, m}\right\|_{2}^{2}=\sum_{k=0}^{m} a_{h_{d}, k}^{2} \leq \sum_{k=0}^{\infty} a_{h_{d}, k}^{2}=\left\|h_{d}\right\|^{2}<\infty
$$

and

$$
\left\|\overrightarrow{\boldsymbol{a}}_{h_{c}, m}\right\|_{2}^{2}=\sum_{k=0}^{m} a_{h_{c}, k}^{2} \leq \sum_{k=0}^{\infty} a_{h_{c}, k}^{2}=\left\|h_{c}\right\|^{2}<\infty .
$$

This completes the proof.

Lemma 10. Suppose that Conditions 1, 3, and 4(2) hold true. Then for each $m \geq 1$, we have

$$
\left\|\widehat{\vec{a}}_{h_{d}, m}-\overrightarrow{\boldsymbol{a}}_{h_{d}, m}\right\|_{2}^{2}=O_{p}\left((n \Delta)^{-1}+\Delta^{2}\right) .
$$


Discrete Dynamics in Nature and Society

7

Proof. First, we have

$$
\begin{aligned}
& \left\|\widehat{\overrightarrow{\boldsymbol{a}}}_{h_{d}, m}-\overrightarrow{\boldsymbol{a}}_{h_{d}, m}\right\|_{2}^{2}=\sum_{k=0}^{m}\left(\widehat{a}_{h_{d}, k}-a_{h_{d}, k}\right)^{2} \\
& =\sum_{k=0}^{m}\left(\int_{0}^{\infty}\left(e^{-\hat{\theta} u}-e^{-\theta u}\right) \psi_{k}(u) d u\right)^{2} \\
& \leq \sum_{k=0}^{\infty}\left(\int_{0}^{\infty}\left(e^{-\hat{\theta} u}-e^{-\theta u}\right) \psi_{k}(u) d u\right)^{2} \\
& =\int_{0}^{\infty}\left(e^{-\hat{\theta} u}-e^{-\theta u}\right)^{2} d u .
\end{aligned}
$$

Using the mean value theorem we obtain $e^{-\hat{\theta} u}-e^{-\theta u}=-(\widehat{\theta}-$ $\theta) u e^{-\theta^{*} u}$, where $\theta^{*}$ is a random number between $\widehat{\theta}$ and $\theta$. Then we have

$$
\begin{aligned}
\left\|\widehat{\vec{a}}_{h_{d}, m}-\overrightarrow{\boldsymbol{a}}_{h_{d}, m}\right\|_{2}^{2} & \leq(\widehat{\theta}-\theta)^{2} \int_{0}^{\infty} u^{2} e^{-z \theta^{*} u} d u \\
& =\frac{(\widehat{\theta}-\theta)^{2}}{\psi\left(\theta^{*}\right)^{3}} .
\end{aligned}
$$

Next, since $\widehat{\sigma}^{2}-\sigma^{2}=O_{p}\left((n \Delta)^{-1 / 2}\right)$ and Lemma 7, then we have

$$
\begin{aligned}
\widehat{\theta}-\theta & =\frac{2 c}{\widehat{\sigma}^{2}}+\widehat{\rho}-\left(\frac{2 c}{\sigma^{2}}+\rho\right) \\
& =2 c\left(\frac{1}{\widehat{\sigma}^{2}}-\frac{1}{\sigma^{2}}\right)+(\widehat{\rho}-\rho) \\
& =O_{p}\left((n \Delta)^{-1 / 2}+\Delta\right)
\end{aligned}
$$

and $\theta^{*}$ converges to $\theta$ in probability. Hence, (72) leads to (70).

Lemma 11. Suppose that Conditions 1, 3, 4(2 +4$), 4\left(\kappa^{\prime}+2\right)$, 5, and 6 hold true. Then we have

$$
\begin{aligned}
\left\|\widehat{\overrightarrow{\boldsymbol{a}}}_{h_{c}, m}-\overrightarrow{\boldsymbol{a}}_{h_{c}, m}\right\|_{2}^{2}= & O_{p}\left(m\left((n \Delta)^{-1}+\Delta^{2}\right)\right) \\
& +o\left(m \Delta^{2 \alpha}\right) .
\end{aligned}
$$

Proof. First, we have

$$
\begin{aligned}
& \left\|\widehat{\overrightarrow{\boldsymbol{a}}}_{h_{c}, m}-\overrightarrow{\boldsymbol{a}}_{h_{c}, m}\right\|_{2}^{2}=\sum_{k=0}^{m}\left(\widehat{a}_{h_{c}, k}-a_{h_{c}, k}\right)^{2} \\
& \quad=\sum_{k=0}^{m}\left(\frac{2}{\widehat{\sigma}^{2}} \frac{1}{n \Delta} \sum_{j=1}^{n} R_{k}\left(Z_{j}, \widehat{\rho}, \widehat{\theta}\right)\right. \\
& -\frac{2}{\widehat{\sigma}^{2}} \int_{0}^{\infty} R_{k}(z, \rho, \theta) \nu(z) d z \\
& \left.+\left(\frac{2}{\widehat{\sigma}^{2}}-\frac{2}{\sigma^{2}}\right) \int_{0}^{\infty} R_{k}(z, \rho, \theta) v(z) d z\right)^{2} \leq \frac{8}{\widehat{\sigma}^{4}}
\end{aligned}
$$

$$
\begin{aligned}
& \cdot \sum_{k=0}^{m}\left(\frac{1}{n \Delta} \sum_{j=1}^{n} R_{k}\left(Z_{j}, \hat{\rho}, \widehat{\theta}\right)\right. \\
& \left.-\int_{0}^{\infty} R_{k}(z, \rho, \theta) \nu(z) d z\right)^{2}+8\left(\frac{1}{\widehat{\sigma}^{2}}-\frac{1}{\sigma^{2}}\right)^{2} \\
& \cdot \sum_{k=0}^{m}\left(\int_{0}^{\infty} R_{k}(z, \rho, \theta) \nu(z) d z\right)^{2}=\frac{8}{\widehat{\sigma}^{4}} \\
& \cdot \sum_{k=0}^{m}\left(\frac{1}{n \Delta} \sum_{j=1}^{n} R_{k}(Z, \hat{\rho}, \widehat{\theta})\right. \\
& \left.-\int_{0}^{\infty} R_{k}(z, \rho, \theta) \nu(z) d z\right)^{2}+2\left(\frac{1}{\widehat{\sigma}^{2}}-\frac{1}{\sigma^{2}}\right)^{2} \\
& \cdot \sigma^{4} \sum_{k=0}^{m} a_{h_{c}, k}^{2} .
\end{aligned}
$$

Since $\widehat{\sigma}^{2}-\sigma^{2}=O_{p}\left((n \Delta)^{-1 / 2}\right)$ and $\sum_{k=0}^{m} a_{h_{c}, k}^{2} \leq \sum_{k=0}^{\infty} a_{h_{c}, k}^{2}=$ $\left\|h_{c}\right\|^{2}<\infty$, we have

$$
2\left(\frac{1}{\widehat{\sigma}^{2}}-\frac{1}{\sigma^{2}}\right)^{2} \sigma^{4} \cdot \sum_{k=0}^{m} a_{h_{c}, k}^{2}=O_{p}\left((n \Delta)^{-1}\right) .
$$

Next, we can write

$$
\begin{aligned}
& \frac{1}{n \Delta} \sum_{j=1}^{n} R_{k}\left(Z_{j}, \hat{\rho}, \widehat{\theta}\right)-\int_{0}^{\infty} R_{k}(z, \rho, \theta) v(z) d z \\
& \quad=\mathrm{I}_{k, 1}+\mathrm{I}_{k, 2}+\mathrm{I}_{k, 3}+\mathrm{I}_{k, 4},
\end{aligned}
$$

where

$$
\begin{aligned}
& \mathrm{I}_{k, 1}=\frac{1}{n \Delta} \sum_{j=1}^{n}\left[R_{k}\left(Z_{j}, \hat{\rho}, \widehat{\theta}\right)-R_{k}\left(Z_{j}, \rho, \widehat{\theta}\right)\right], \\
& \mathrm{I}_{k, 2}=\frac{1}{n \Delta} \sum_{j=1}^{n}\left[R_{k}\left(Z_{j}, \rho, \widehat{\theta}\right)-R_{k}\left(Z_{j}, \rho, \theta\right)\right], \\
& \mathrm{I}_{k, 3}=\frac{1}{n \Delta} \sum_{j=1}^{n} R_{k}\left(Z_{j}, \rho, \theta\right)-\frac{1}{\Delta} E\left[R_{k}\left(Z_{j}, \rho, \theta\right)\right], \\
& \mathrm{I}_{k, 4}=\frac{1}{\Delta} E\left[R_{k}\left(Z_{j}, \rho, \theta\right)\right]-\int_{0}^{\infty} R_{k}(z, \rho, \theta) \nu(z) d z .
\end{aligned}
$$

Then using Jensen inequality we have

$$
\begin{aligned}
& \sum_{k=0}^{m}\left(\frac{1}{n \Delta} \sum_{j=1}^{n} R_{k}\left(Z_{j}, \hat{\rho}, \hat{\theta}\right)-\int_{0}^{\infty} R_{k}(z, \rho, \theta) v(z) d z\right)^{2} \\
& \quad=\sum_{k=0}^{m}\left(\mathrm{I}_{k, 1}+\mathrm{I}_{k, 2}+\mathrm{I}_{k, 3}+\mathrm{I}_{k, 4}\right)^{2} \\
& \quad \leq 4 \sum_{k=0}^{m}\left[\mathrm{I}_{k, 1}^{2}+\mathrm{I}_{k, 2}^{2}+\mathrm{I}_{k, 3}^{2}+\mathrm{I}_{k, 4}^{2}\right] .
\end{aligned}
$$


For $\mathrm{I}_{k, 1}$, using mean value theory we obtain

$$
\begin{aligned}
\mathrm{I}_{k, 1} & =\frac{1}{n \Delta} \sum_{j=1}^{n} \int_{0}^{Z_{j}} \int_{0}^{z} \int_{y}^{\infty} e^{-\hat{\theta}(u-y)}\left[e^{-\hat{\rho}(z-y)}-e^{-\rho(z-y)}\right] \varphi_{k}(u) w\left(Z_{j}-z\right) d u d y d z \\
& =-\frac{1}{n \Delta} \sum_{j=1}^{n} \int_{0}^{Z_{j}} \int_{0}^{z} \int_{y}^{\infty} e^{-\hat{\theta}(u-y)}(\hat{\rho}-\rho)(z-y) e^{-\rho^{*}(z-y)} \varphi_{k}(u) w\left(Z_{j}-z\right) d u d y d z
\end{aligned}
$$

where $\rho^{*}$ is a random number between $\rho$ and $\widehat{\rho}$. Then using the upper bound $\sup _{x \geq 0}\left|\varphi_{k}(x)\right| \leq \sqrt{2}$ we obtain

$$
\begin{aligned}
& \sup _{k}\left|\mathrm{I}_{k, 1}\right| \leq \sqrt{2}|\widehat{\rho}-\rho| \frac{1}{n \Delta} \\
& \cdot \sum_{j=1}^{n} \int_{0}^{Z_{j}} \int_{0}^{z} \int_{y}^{\infty} e^{-\hat{\theta}(u-y)}(z-y) w\left(Z_{j}-z\right) d u d y d z \\
& \leq C \cdot|\widehat{\rho}-\rho| \\
& \quad \cdot \frac{1}{n \Delta} \sum_{j=1}^{n} \int_{0}^{Z_{j}} \int_{0}^{z} \int_{y}^{\infty} e^{-\widehat{\theta}(u-y)}(z-y)\left(1+Z_{j}-z\right)^{\kappa} d u d y d z \\
& \leq C \cdot|\widehat{\rho}-\rho| \cdot \frac{1}{\hat{\theta}} \\
& \quad \cdot \frac{1}{n \Delta} \sum_{j=1}^{n} \int_{0}^{Z_{j}} \int_{0}^{z}(z \\
& \quad-y)\left(1+Z_{j}-z\right)^{\kappa} d y d z \\
& \leq C \cdot|\widehat{\rho}-\rho| \cdot \frac{1}{\hat{\theta}} \cdot \frac{1}{n \Delta} \sum_{j=1}^{n}\left(1+Z_{j}\right)^{\kappa} Z_{j}^{3} .
\end{aligned}
$$

Since $\widehat{\sigma}^{2} \longrightarrow \sigma^{2}$ and $\hat{\rho} \longrightarrow \rho$ in probability, we have $\widehat{\theta} \longrightarrow \theta>0$ in probability, leading to $\hat{\theta}=O_{p}(1)$. Using Markov inequality we can obtain $(1 / n \Delta) \sum_{j=1}^{n}\left(1+Z_{j}\right)^{\kappa} Z_{j}^{3}=$ $O_{p}(1)$ under Condition $4(\kappa+3)$. Hence, by Lemma 7 we obtain

$$
\begin{aligned}
\sup _{k}\left|I_{k, 1}\right| & =O_{p}\left((n \Delta)^{-1 / 2}+\Delta\right) \cdot O_{p}(1) \cdot O_{p}(1) \\
& =O_{p}\left((n \Delta)^{-1 / 2}+\Delta\right)
\end{aligned}
$$

which yields

$$
\sum_{k=0}^{m} \mathrm{I}_{k, 1}^{2}=O_{p}\left(m\left((n \Delta)^{-1}+\Delta^{2}\right)\right)
$$

For $\mathrm{I}_{k, 2}$, using mean value theory we have

$$
\begin{aligned}
\mathrm{I}_{k, 2} & =\frac{1}{n \Delta} \sum_{j=1}^{n}\left[R_{k}\left(Z_{j}, \rho, \widehat{\theta}\right)-R_{k}\left(Z_{j}, \rho, \theta\right)\right]=\frac{1}{n \Delta} \sum_{j=1}^{n} \int_{0}^{Z_{j}} \int_{0}^{z} \int_{y}^{\infty}\left[e^{-\hat{\theta}(u-y)}-e^{-\theta(u-y)}\right] e^{-\rho(z-y)} \varphi_{k}(u) w\left(Z_{j}-z\right) d u d y d z \\
& =-\frac{1}{n \Delta} \sum_{j=1}^{n} \int_{0}^{Z_{j}} \int_{0}^{z} \int_{y}^{\infty}(\widehat{\theta}-\theta)(u-y) e^{-\theta^{*}(u-y)} e^{-\rho(z-y)} \varphi_{k}(u) w\left(Z_{j}-z\right) d u d y d z
\end{aligned}
$$

where $\theta^{*}$ is a random number between $\hat{\theta}$ and $\theta$. Then we have

$$
\begin{gathered}
\sup _{k}\left|\mathrm{I}_{k, 2}\right| \leq \sqrt{2}|\hat{\theta}-\theta| \\
\cdot \frac{1}{n \Delta} \sum_{j=1}^{n} \int_{0}^{Z_{j}} \int_{0}^{z} \int_{y}^{\infty}(u-y) e^{-\theta^{*}(u-y)} w\left(Z_{j}-z\right) d u d y d z \\
=\sqrt{2}|\hat{\theta}-\theta| \cdot \frac{1}{\left(\theta^{*}\right)^{2}} \frac{1}{n \Delta} \sum_{j=1}^{n} \int_{0}^{Z_{j}} \int_{0}^{z} w\left(Z_{j}-z\right) d y d z \\
\leq C \cdot|\hat{\theta}-\theta| \cdot \frac{1}{\left(\theta^{*}\right)^{2}} \frac{1}{n \Delta} \sum_{j=1}^{n} \int_{0}^{Z_{j}} \int_{0}^{z}\left(1+Z_{j}-z\right)^{\kappa} d y d z
\end{gathered}
$$

$$
\leq C \cdot|\widehat{\theta}-\theta| \cdot \frac{1}{\left(\theta^{*}\right)^{2}} \frac{1}{n \Delta} \sum_{j=1}^{n}\left(1+Z_{j}\right)^{\kappa} Z_{j}^{2},
$$

which together with (74) and Condition $4(\kappa+2)$ gives $\sup _{k}\left|\mathrm{I}_{k, 2}\right|=O_{p}\left((n \Delta)^{-1 / 2}+\Delta\right)$. Then we obtain

$$
\sum_{k=0}^{m} \mathrm{I}_{k, 2}^{2}=O_{p}\left(m\left((n \Delta)^{-1}+\Delta^{2}\right)\right) .
$$

For $\mathrm{I}_{k, 3}$ we have

$$
E\left\{\sum_{k=0}^{m} \mathrm{I}_{k, 3}^{2}\right\}=\sum_{k=0}^{m} E\left[\mathrm{I}_{k, 3}^{2}\right]=\sum_{k=0}^{m} \operatorname{var} t\left(\mathrm{I}_{k, 3}\right)
$$




$$
\begin{aligned}
& =\sum_{k=0}^{m} \frac{1}{n \Delta^{2}} \operatorname{var}\left(R_{k}\left(Z_{1}, \rho, \theta\right)\right) \\
& \leq \frac{1}{n \Delta^{2}} \sum_{k=0}^{m} E\left[\left(R_{k}\left(Z_{1}, \rho, \theta\right)\right)^{2}\right] .
\end{aligned}
$$

Because

$$
\begin{gathered}
\left|R_{k}\left(Z_{1}, \rho, \theta\right)\right| \leq \int_{0}^{Z_{1}} \int_{0}^{z} \int_{y}^{\infty} e^{-\theta(u-y)} e^{-\rho(z-y)}\left|\varphi_{k}(u)\right| \\
\cdot w\left(Z_{j}-z\right) d u d y d z \leq \frac{\sqrt{2}}{\theta} \int_{0}^{Z_{1}} \int_{0}^{z} w\left(Z_{j}\right. \\
-z) d y d z \leq C\left(1+Z_{1}\right)^{\kappa} Z_{1}^{2},
\end{gathered}
$$

we have

$$
\begin{aligned}
E\left\{\sum_{k=0}^{m} \mathrm{I}_{k, 3}^{2}\right\} & \leq C \cdot \frac{1}{n \Delta^{2}} \sum_{k=0}^{m} E\left[\left(1+Z_{1}\right)^{2 \kappa} \cdot Z_{1}^{4}\right] \\
& =C m(n \Delta)^{-1}
\end{aligned}
$$

due to Condition $4(2 \kappa+4)$. Then Markov inequality gives

$$
\sum_{k=0}^{m} \mathrm{I}_{k, 3}^{2}=O_{p}\left(m(n \Delta)^{-1}\right) .
$$

For $\mathrm{I}_{k, 4}$, note that

$$
\sup _{k \geq 0}\left|R_{k}(x, \rho, \theta)\right| \leq C \cdot(1+x)^{\kappa} x^{2}
$$

and

$$
\begin{aligned}
& \sup _{k \geq 0}\left|\frac{\partial}{\partial x} R_{k}(x, \rho, \theta)\right| \\
& =\sup _{k \geq 0} \mid \int_{0}^{x} \int_{y}^{\infty} e^{-\theta(u-y)} e^{-\rho(z-y)} \varphi_{k}(u) \\
& \cdot w(0) d u d y+\int_{0}^{x} \int_{0}^{z} \int_{y}^{\infty} e^{-\theta(u-y)} e^{-\rho(z-y)} \varphi_{k}(u) \\
& \cdot w^{\prime}(x-z) d u d y d z \mid \leq C x \\
& +C \int_{0}^{x} \int_{0}^{z} \int_{y}^{\infty} e^{-\theta(u-y)}\left|w^{\prime}(x-z)\right| d u d y d z \\
& \leq C x+C \int_{0}^{x} \int_{0}^{z}(1+x-z)^{\kappa^{\prime}} d y d z \leq C x \\
& +C(1+x)^{\kappa^{\prime}} x^{2} .
\end{aligned}
$$

Then by Lemma B.1 in Shimizu and Zhang [45] we have $\sup _{k \geq 0}\left|\mathrm{I}_{k, 4}\right|=o\left(\Delta^{\alpha}\right)$, which yields

$$
\sum_{k=0}^{m} \mathrm{I}_{k, 4}^{2}=o\left(m \Delta^{2 \alpha}\right) .
$$

By (79), (83), (86), (90), and (93) we have

$$
\begin{aligned}
& \sum_{k=0}^{m}\left(\frac{1}{n \Delta} \sum_{j=1}^{n} R_{k}\left(Z_{j}, \hat{\rho}, \widehat{\theta}\right)-\int_{0}^{\infty} R_{k}(z, \rho, \theta) v(z) d z\right)^{2} \\
& =O_{p}\left(m\left((n \Delta)^{-1}+\Delta^{2}\right)+o\left(m \Delta^{2 \alpha}\right)\right)
\end{aligned}
$$

which together with (75) and (76) gives (74).

Lemma 12. Suppose that Conditions 1, 3, 4(2), and 5 hold true. Then we have

$$
\left\|\boldsymbol{A}_{m}-\widehat{\boldsymbol{A}}_{m}\right\|_{F}^{2}=O_{p}\left(m^{2}\left((n \Delta)^{-1}+\Delta^{2}\right)\right)+o\left(m^{2} \Delta^{2 \alpha}\right) .
$$

Furthermore, if $m^{2}=o\left((n \Delta)+\Delta^{-2 \alpha}\right)$, then we have

$$
\begin{aligned}
\left\|\boldsymbol{A}_{m}^{-1}-\widehat{\boldsymbol{A}}_{m}^{-1}\right\|_{2}= & O_{p}\left(m^{2}\left((n \Delta)^{-1}+\Delta^{2}\right)\right) \\
& +o\left(m^{2} \Delta^{2 \alpha}\right) .
\end{aligned}
$$

Proof. By the definitions of $\boldsymbol{A}_{m}$ and $\widehat{\boldsymbol{A}}_{m}$ we have

$$
\begin{aligned}
& \left\|\boldsymbol{A}_{m}-\widehat{\boldsymbol{A}}_{m}\right\|_{F}^{2}=\sum_{k=0}^{m} \sum_{l=0}^{k} 2^{-1}\left(\left[\widehat{a}_{g, k-l}-a_{g, k-l}\right]^{2}\right. \\
& \left.+\left[\widehat{a}_{g, k-l-1}-a_{g, k-l-1}\right]^{2}\right) \leq \sum_{k=0}^{m} \sum_{l=0}^{k}\left(\left[\widehat{a}_{g, k-l}-a_{g, k-l}\right]^{2}\right. \\
& \left.+\left[\widehat{a}_{g, k-l-1}-a_{g, k-l-1}\right]^{2}\right) \leq 2 \sum_{k=0}^{m} \sum_{l=0}^{k}\left[\widehat{a}_{g, k-l}-a_{g, k-l}\right]^{2} \\
& \quad \leq 2(m+1) \sum_{k=0}^{m}\left[\widehat{a}_{g, k}-a_{g, k}\right]^{2} .
\end{aligned}
$$

For each $k$, we have

$$
\begin{aligned}
& \widehat{a}_{g, k}-a_{g, k}=\frac{2}{\widehat{\sigma}^{2}} \frac{1}{n \Delta} \sum_{j=1}^{n} Q_{k}\left(Z_{j}, \widehat{\rho}, \widehat{\theta}\right)-\frac{2}{\sigma^{2}} \\
& \cdot \int_{0}^{\infty} Q_{k}(z, \rho, \theta) \nu(z) d z \\
& =\frac{2}{\widehat{\sigma}^{2}}\left\{\frac{1}{n \Delta} \sum_{j=1}^{n} Q_{k}\left(Z_{j}, \widehat{\rho}, \widehat{\theta}\right)\right. \\
& \left.-\int_{0}^{\infty} Q_{k}(z, \rho, \theta) \nu(z) d z\right\}+\left(\frac{1}{\widehat{\sigma}^{2}}-\frac{1}{\sigma^{2}}\right) \sigma^{2} a_{g, k}
\end{aligned}
$$


Then we obtain

$$
\begin{aligned}
& \left\|\boldsymbol{A}_{m}-\widehat{\boldsymbol{A}}_{m}\right\|_{F}^{2} \leq 2(m+1) \\
& \cdot \sum_{k=0}^{m}\left(\frac { 2 } { \widehat { \sigma } ^ { 2 } } \left\{\frac{1}{n \Delta} \sum_{j=1}^{n} Q_{k}\left(Z_{j}, \hat{\rho}, \widehat{\theta}\right)\right.\right. \\
& \left.-\int_{0}^{\infty} Q_{k}(z, \rho, \theta) v(z) d z\right\}+\left(\frac{1}{\widehat{\sigma}^{2}}-\frac{1}{\sigma^{2}}\right) \\
& \left.\cdot \sigma^{2} a_{g, k}\right)^{2} \leq \frac{16}{\widehat{\sigma}^{4}}(m+1) \sum_{k=0}^{m}\left(\frac{1}{n \Delta} \sum_{j=1}^{n} Q_{k}\left(Z_{j}, \widehat{\rho}, \widehat{\theta}\right)\right. \\
& \left.-\int_{0}^{\infty} Q_{k}(z, \rho, \theta) v(z) d z\right)^{2}+2\left(\frac{1}{\widehat{\sigma}^{2}}-\frac{1}{\sigma^{2}}\right)^{2} \\
& \cdot \sigma^{4}(m+1) \sum_{k=0}^{m} a_{g, k}^{2} .
\end{aligned}
$$

Note that

$$
\begin{aligned}
& \left(\frac{1}{\widehat{\sigma}^{2}}-\frac{1}{\sigma^{2}}\right)^{2} \sigma^{4}(m+1) \sum_{k=0}^{m} a_{g, k}^{2} \\
& \quad \leq\left(\frac{1}{\widehat{\sigma}^{2}}-\frac{1}{\sigma^{2}}\right)^{2} \cdot \sigma^{4}(m+1) \sum_{k=0}^{\infty} a_{g, k}^{2} \\
& \quad=\left(\frac{1}{\widehat{\sigma}^{2}}-\frac{1}{\sigma^{2}}\right)^{2} \sigma^{4}(m+1)\|g\|^{2}=O_{p}\left(m(n \Delta)^{-1}\right)
\end{aligned}
$$

due to $\widehat{\sigma}^{2}-\sigma^{2}=O_{p}\left((n \Delta)^{-1 / 2}\right)$.

For each $k$, we can write

$$
\begin{aligned}
& \frac{1}{n \Delta} \sum_{j=1}^{n} Q_{k}(Z, \hat{\rho}, \widehat{\theta})-\int_{0}^{\infty} Q_{k}(z, \rho, \theta) \nu(z) d z \\
& \quad=\mathrm{II}_{k, 1}+\mathrm{II}_{k, 2}+\mathrm{II}_{k, 3}+\mathrm{II}_{k, 4},
\end{aligned}
$$

where

$$
\begin{aligned}
& \mathrm{II}_{k, 1}=\frac{1}{n \Delta} \sum_{j=1}^{n}\left[Q_{k}\left(Z_{j}, \hat{\rho}, \widehat{\theta}\right)-Q_{k}\left(Z_{j}, \rho, \widehat{\theta}\right)\right], \\
& \mathrm{II}_{k, 2}=\frac{1}{n \Delta} \sum_{j=1}^{n}\left[Q_{k}\left(Z_{j}, \rho, \widehat{\theta}\right)-Q_{k}\left(Z_{j}, \rho, \theta\right)\right], \\
& \mathrm{II}_{k, 3}=\frac{1}{n \Delta} \sum_{j=1}^{n} Q_{k}\left(Z_{j}, \rho, \theta\right)-\frac{1}{\Delta} E\left[Q_{k}\left(Z_{1}, \rho, \theta\right)\right], \\
& \mathrm{II}_{k, 4}=\frac{1}{\Delta} E\left[Q_{k}\left(Z_{1}, \rho, \theta\right)\right]-\int_{0}^{\infty} Q_{k}(z, \rho, \theta) \nu(z) d z .
\end{aligned}
$$

Then using Jensen inequality, we get

$$
\begin{aligned}
& \sum_{k=0}^{m}\left(\frac{1}{n \Delta} \sum_{j=1}^{n} Q_{k}\left(Z_{j}, \widehat{\rho}, \widehat{\theta}\right)\right. \\
& \left.\quad-\int_{0}^{\infty} Q_{k}(z, \rho, \theta) \nu(z) d z\right)^{2}=\sum_{k=0}^{m}\left(\mathrm{II}_{k, 1}+\mathrm{II}_{k, 2}\right. \\
& \left.\quad+\mathrm{II}_{k, 3}+\mathrm{II}_{k, 4}\right)^{2} \leq 4 \sum_{k=0}^{m}\left[\mathrm{II}_{k, 1}^{2}+\mathrm{II}_{k, 2}^{2}+\mathrm{II}_{k, 3}^{2}+\mathrm{II}_{k, 4}^{2}\right] .
\end{aligned}
$$

Using the similar method as in the proof of Lemma 12, we can obtain

$$
\sum_{k=0}^{m} \mathrm{II}_{k, l}^{2}=O_{p}\left(m\left((n \Delta)^{-1}+\Delta^{2}\right)\right), \quad l=1,2,3,
$$

and $\sum_{k=0}^{m} \mathrm{II}_{k, 4}^{2}=o\left(m\left(\Delta^{2 \alpha}\right)\right)$. Hence, we have

$$
\begin{aligned}
& \frac{16}{\widehat{\sigma}^{4}}(m+1) \sum_{k=0}^{m}\left(\frac{1}{n \Delta} \sum_{j=1}^{n} Q_{k}\left(Z_{j}, \widehat{\rho}, \widehat{\theta}\right)\right. \\
& \left.\quad-\int_{0}^{\infty} Q_{k}(z, \rho, \theta) \nu(z) d z\right)^{2}=O_{p}\left(m ^ { 2 } \left((n \Delta)^{-1}\right.\right. \\
& \left.\left.+\Delta^{2}\right)\right)+o\left(m^{2} \Delta^{2 \alpha}\right) .
\end{aligned}
$$

Finally, by (99), (100), and (105) we obtain (95).

Now we use Theorem B.1 in Zhang and Su [39] to prove this result. First, note that $\widehat{A}_{m}=A_{m}+\widehat{A}_{m}-A_{m}$ and $A_{m}$ is nonsingular. By Lemma 8 and formula (95) we have

$$
\begin{aligned}
\left\|A_{m}^{-1}\left(\widehat{A}_{m}-A_{m}\right)\right\|_{2} & \leq\left\|A_{m}^{-1}\right\|_{2} \cdot\left\|\widehat{A}_{m}-A_{m}\right\|_{2} \\
& \leq C \cdot\left\|\widehat{A}_{m}-A_{m}\right\|_{F}=o_{p}(1)
\end{aligned}
$$

under condition $m^{2}=o\left((n \Delta)+\Delta^{-2 \alpha}\right)$. Hence, by Lemma B.1 in Zhang and $\mathrm{Su}$ [39] we obtain

$$
\begin{aligned}
\left\|\boldsymbol{A}_{m}^{-1}-\widehat{\boldsymbol{A}}_{m}^{-1}\right\|_{2} & \leq \frac{\left\|\boldsymbol{A}_{m}-\widehat{\boldsymbol{A}}_{m}\right\|_{2} \cdot\left\|\boldsymbol{A}_{m}^{-1}\right\|_{2}^{2}}{1-\left\|\boldsymbol{A}_{m}^{-1}\left(\widehat{\boldsymbol{A}}_{m}-\boldsymbol{A}_{m}\right)\right\|_{2}} \\
& \leq C \frac{\left\|\boldsymbol{A}_{m}-\widehat{\boldsymbol{A}}_{m}\right\|_{F}}{1-\left\|\boldsymbol{A}_{m}^{-1}\left(\widehat{\boldsymbol{A}}_{m}-\boldsymbol{A}_{m}\right)\right\|_{2}} \\
& =O_{p}\left(m\left((n \Delta)^{-1 / 2}+\Delta\right)\right)+o\left(m \Delta^{\alpha}\right) .
\end{aligned}
$$

This completes the proof.

The main results of this section are given below. 
Theorem 13. Suppose that Conditions 1, 2, 3, $4(2 \kappa+4), 4\left(\kappa^{\prime}+2\right), 5,6$, and $m^{2}=o\left((n \Delta)+\Delta^{-2 \alpha}\right)$ hold true. Then we have

$$
\begin{aligned}
\left\|\hat{\phi}_{d, m}-\phi_{d, m}\right\|^{2}= & O_{p}\left(m^{2}\left((n \Delta)^{-1}+\Delta^{2}\right)\right) \\
& +o\left(m^{2} \Delta^{2 \alpha}\right), \\
\left\|\hat{\phi}_{c, m}-\phi_{c, m}\right\|^{2}= & O_{p}\left(m^{2}\left((n \Delta)^{-1}+\Delta^{2}\right)\right) \\
& +o\left(m^{2} \Delta^{2 \alpha}\right) .
\end{aligned}
$$

Furthermore, if $\phi_{d}, \phi_{c} \in W\left(\mathbb{R}_{+}, r, B\right)$, then we have

$$
\begin{aligned}
\left\|\widehat{\phi}_{d, m}-\phi_{d}\right\|^{2}= & O\left(m^{-r}\right)+O_{p}\left(m^{2}\left((n \Delta)^{-1}+\Delta^{2}\right)\right) \\
& +o\left(m^{2} \Delta^{2 \alpha}\right), \\
\left\|\widehat{\phi}_{c, m}-\phi_{c}\right\|^{2}= & O\left(m^{-r}\right)+O_{p}\left(m^{2}\left((n \Delta)^{-1}+\Delta^{2}\right)\right) \\
& +o\left(m^{2} \Delta^{2 \alpha}\right) .
\end{aligned}
$$

Proof. The above results follow from formulae (58), (60), and (61) and Lemmas 9-12.

\section{Simulation Studies}

In this section, we present some numerical examples to show the effectiveness of our estimator when the sample size is finite. We suppose that the risk model is a compound Poisson process perturbed by a diffusion, where we set the premium rate $c=15$ and diffusion volatility parameter $\sigma^{2}=6$ and the intensity of Poisson claim number process is 10 . In addition, we assume that the claim size distribution is an exponential distribution with mean 1 and the $\operatorname{Erlang}(2,2)$ distribution. Then the Lévy densities are given by

$$
v(d x)=10 e^{-x} d x, \quad x>0
$$

and

$$
v(d x)=40 x e^{-2 x} d x, \quad x>0
$$

respectively.

We shall estimate the ruin probability and the Laplace transform of ruin time, where the penalty function $w \equiv$ 1. For ruin probability, the discount factor $\delta=0$. For Laplace transform of ruin time, the discount factor $\delta=$ 0.01 . The reference values of the Gerber-Shiu function can be explicit obtained by Laplace inversion transform. For ruin probability, when the claim amount obeys the exponential distribution, we obtain

$$
\begin{aligned}
\phi_{d}(u)=0.8693 * e^{-5.7080 * u}+0.1307 * e^{-0.2920 * u}, & \\
u & \geq 0, \\
\phi_{c}(u)=-0.6155 * e^{-5.7080 * u}+0.6155 * e^{-0.2920 * u}, & \\
u & \geq 0 ;
\end{aligned}
$$

when the claim amount obeys the $\operatorname{Erlang}(2,2)$ distributed, we obtain

$$
\begin{aligned}
\phi_{d}(u)= & 1.0531 * e^{-5.4039 * u}-0.2370 * e^{-3.2120 * u} \\
& +0.1839 * e^{-0.3841 * u}, \quad u \geq 0 \\
\phi_{c}(u)= & -0.7283 * e^{-5.4039 * u}+0.1140 * e^{-3.2120 * u} \\
& +0.6142 * e^{-0.3841 * u}, \quad u \geq 0 .
\end{aligned}
$$

For the Laplace transform of ruin time, when the claim amount obeys the exponential distribution, we obtain

$$
\begin{aligned}
\phi_{d}(u)=0.8695 * e^{-5.7085 * u}+0.1305 * e^{-0.2935 * u}, & \\
u & \geq 0, \\
\phi_{c}(u)=-0.6143 * e^{-5.7085 * u}+0.6144 * e^{-0.2935 * u}, & \\
u & \geq 0 ;
\end{aligned}
$$

when the claim amount obeys the $\operatorname{Erlang}(2,2)$ distributed, we obtain

$$
\begin{aligned}
\phi_{d}(u)= & 1.0532 * e^{-5.4045 * u}-0.2370 * e^{-3.2118 * u} \\
& +0.1837 * e^{-0.3857 * u}, \quad u \geq 0 \\
\phi_{c}(u)= & -0.7271 * e^{-5.4045 * u}+0.1135 * e^{-3.2118 * u} \\
& +0.6136 * e^{-0.3857 * u}, \quad u \geq 0 .
\end{aligned}
$$

In the sequel, we shall set $(n, \Delta)=(1000,0.02),(2500$, $0.01),(5000,0.01)$, where $n \Delta=20,25,50$. The truncate parameter is set to be $m=9$. First, let us consider the case where the claim amount is exponentially distributed. For $(n, \Delta)=(2500,0.01)$, in Figures 1 and 2 we plot 30 consecutive estimates on the same picture together with the true GerberShiu functions to show variability bands and illustrate the stability of the procedures. We find that the estimates in the beam are very close to each other and close to the true GerberShiu functions.

To measure the performance of the estimate, we compute the integrated mean-square error (IMSE) based on 300 experiments, which are computed by

$$
\begin{aligned}
& \frac{1}{300} \sum_{j=1}^{300} \int_{0}^{30}\left(\phi_{d}(u)-\widehat{\phi}_{d, j}(u)\right)^{2} d u, \\
& \frac{1}{300} \sum_{j=1}^{300} \int_{0}^{30}\left(\phi_{c}(u)-\widehat{\phi}_{c, j}(u)\right)^{2} d u,
\end{aligned}
$$

where $\widehat{\phi}_{d, j}$ and $\widehat{\phi}_{c, j}$ denote the $j$-th estimated function. Note that the above integrals are computed on a finite domain $[0,30]$, because both the reference value and the estimator are very close to zero as $u$ increases. We give some values of IMSEs in Table 1, where we can easily see that the IMSEs decrease as $n \Delta$ increases for each Gerber-Shiu functions. 

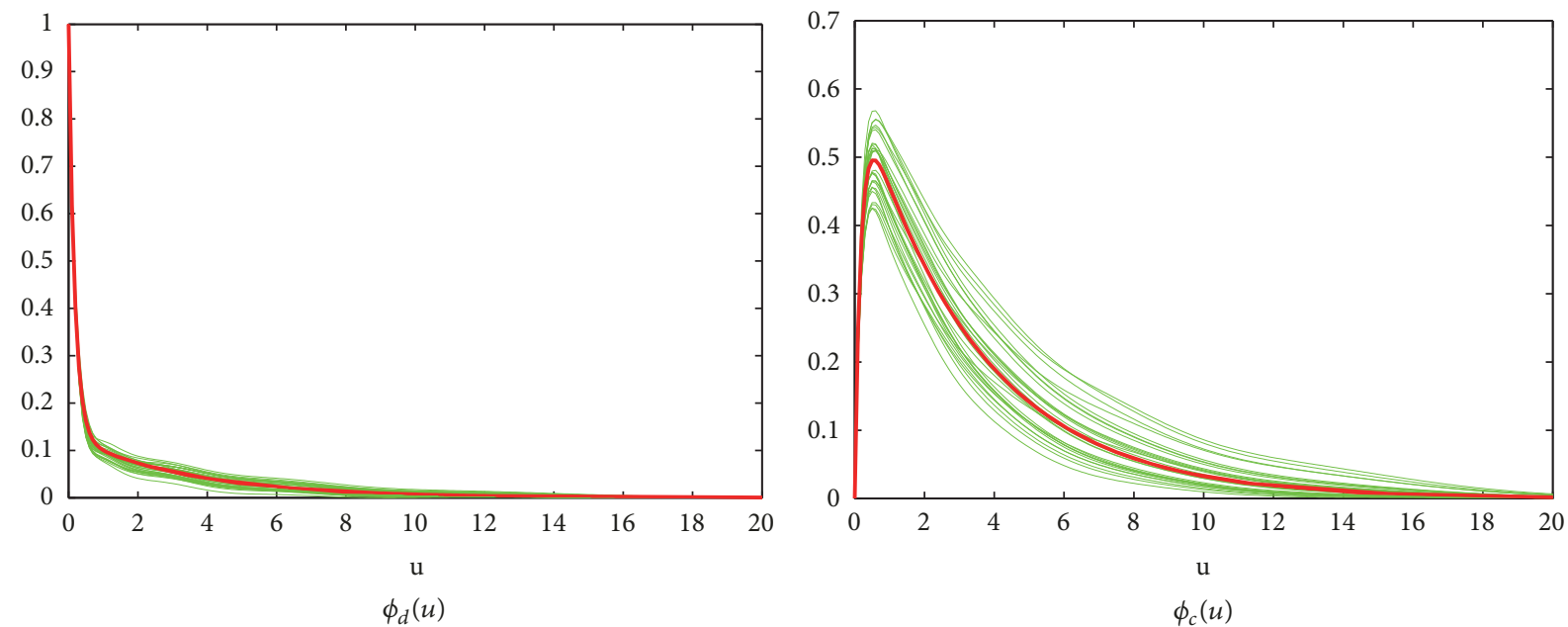

FIGURE 1: Estimating the ruin probability for exponential claim sizes, true curve (red curve) and 30 estimated curves (green curves).

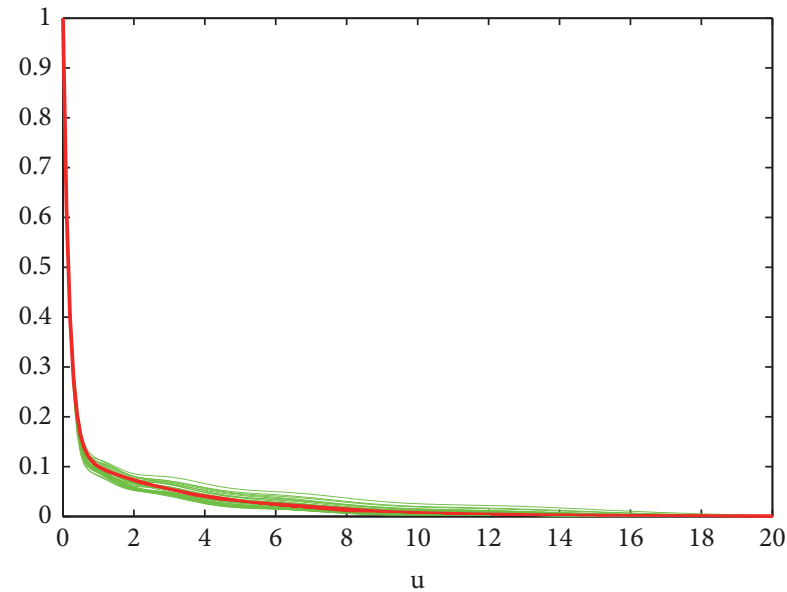

$\phi_{d}(u)$

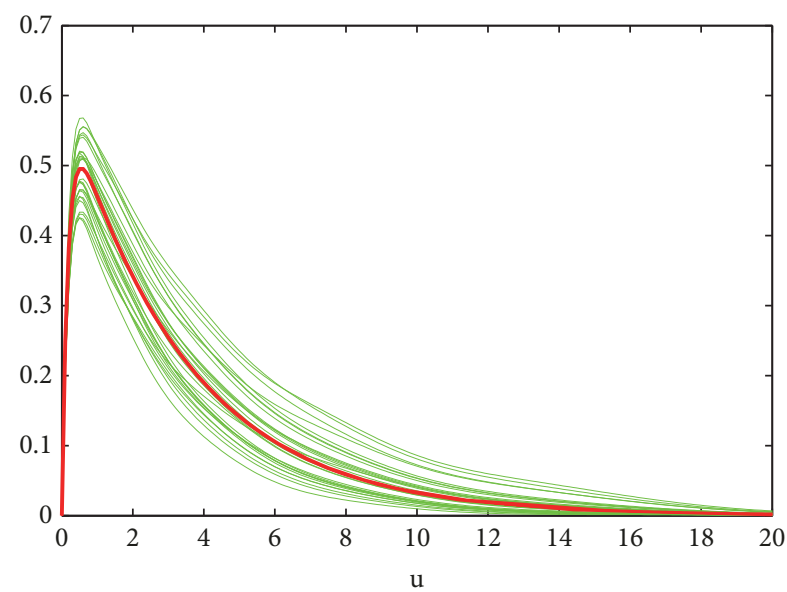

$\phi_{c}(u)$

FIGURE 2: Estimating the Laplace transform of ruin time for exponential claim sizes, true curve (red curve) and 30 estimated curves (green curves).

TABLE 1: IMSEs $\times 100$.

\begin{tabular}{|c|c|c|c|c|c|}
\hline & \multirow[t]{2}{*}{$(n, \Delta)$} & \multicolumn{2}{|c|}{ Ruin probability } & \multicolumn{2}{|c|}{$\begin{array}{l}\text { Laplace transform of } \\
\text { ruin time }\end{array}$} \\
\hline & & $\phi_{d}(u)$ & $\phi_{c}(u)$ & $\phi_{d}(u)$ & $\phi_{c}(u)$ \\
\hline \multirow{3}{*}{ Exponential } & $(1000,0.02)$ & 0.57 & 6.24 & 0.20 & 6.14 \\
\hline & $(2500,0.01)$ & 0.09 & 2.88 & 0.05 & 1.27 \\
\hline & $(5000,0.01)$ & 0.08 & 0.28 & 0.03 & 0.18 \\
\hline \multirow{3}{*}{ Erlang(2,2) } & $(1000,0.02)$ & 0.22 & 1.03 & 0.36 & 2.68 \\
\hline & $(2500,0.01)$ & 0.06 & 0.26 & 0.16 & 1.79 \\
\hline & $(5000,0.01)$ & 0.04 & 0.18 & 0.07 & 0.02 \\
\hline
\end{tabular}




\section{Data Availability}

The data used to support the findings of this study are available from the corresponding author upon request.

\section{Conflicts of Interest}

The authors declare that they have no conflicts of interest.

\section{Acknowledgments}

This research is partially supported by the National Natural Science Foundation of China (Grant Nos. 11301303 and 11501325), the National Social Science Foundation of China (Grant No. 15BJY007), the Taishan Scholars Program of Shandong Province (Grant No. tsqn20161041), the Humanities and Social Sciences Project of the Ministry Education of China (Grant Nos. 16YJC630070 and 19YJA910002), the Natural Science Foundation of Shandong Province (Grant No. ZR2018MG002), the Fostering Project of Dominant Discipline and Talent Team of Shandong Province Higher Education Institutions (Grant No. 1716009), Risk Management and Insurance Research Team of Shandong University of Finance and Economics, the 1251 Talent Cultivation Project of Shandong Jiaotong University, Shandong Jiaotong University 'Climbing' Research Innovation Team Program, and Collaborative Innovation Center Project of the Transformation of New and Old Kinetic Energy and Government Financial Allocation.

\section{References}

[1] H. U. Gerber and E. S. W. Shiu, "On the time value of ruin," North American Actuarial Journal, vol. 2, no. 1, pp. 48-78, 1998.

[2] S. M. Li and J. Garrido, "On ruin for the Erlang(n) risk process," Insurance: Mathematics and Economics, vol. 34, no. 3, pp. 391408, 2004.

[3] S. M. Li and J. Garrido, "The Gerber-Shiu function in a Sparre Andersen risk process perturbed by diffusion," Scandinavian Actuarial Journal, vol. 2005, no. 3, pp. 161-186, 2005.

[4] J. Garrido and M. Morales, "On the expected discounted penalty function for Lévy risk processes," North American Actuarial Journal, vol. 10, no. 4, pp. 196-218, 2006.

[5] E. Biffis and M. Morales, "On a generalization of the GerberShiu function to path-dependent penalties," Insurance: Mathematics and Economics, vol. 46, no. 1, pp. 92-97, 2010.

[6] K. W. Chau, S. C. Yam, and H. Yang, "Fourier-cosine method for Gerber-Shiu functions," Insurance: Mathematics and Economics, vol. 61, pp. 170-180, 2015.

[7] C. C. Yin and C. W. Wang, "Optimality of the barrier strategy in de Finetti's dividend problem for spectrally negative Lévy processes: An alternative approach," Journal of Computational and Applied Mathematics, vol. 233, no. 2, pp. 482-491, 2009.

[8] C. C. Yin and C. W. Wang, "The perturbed compound Poisson risk process with investment and debit interest," Methodology and Computing in Applied Probability, vol. 12, no. 3, pp. 391-413, 2010.

[9] S. Asmussen and H. Albrecher, Ruin Probabilities, vol. 14, World Scientific, Singapore, 2nd edition, 2010.
[10] Y. C. Chi, "Analysis of the expected discounted penalty function for a general jump-diffusion risk model and applications in finance," Insurance: Mathematics and Economics, vol. 46, no. 2, pp. 385-396, 2010.

[11] C. W. Wang, C. C. Yin, and E. Q. Li, "On the classical risk model with credit and debit interests under absolute ruin," Statistics \& Probability Letters, vol. 80, no. 5-6, pp. 427-436, 2010.

[12] Y. C. Chi and X. S. Lin, "On the threshold dividend strategy for a generalized jump-diffusion risk model," Insurance: Mathematics and Economics, vol. 48, no. 3, pp. 326-337, 2011.

[13] X. H. Zhao and C. C. Yin, "The Gerber-Shiu expected discounted penalty function for Lévy insurance risk processes," Acta Mathematicae Applicatae Sinica, English Series, vol. 26, no. 4, pp. 575-586, 2010.

[14] Y. X. Zhao and C. C. Yin, "The expected discounted penalty function under a renewal risk model with stochastic income," Applied Mathematics and Computation, vol. 218, no. 10, pp. 6144-6154, 2012.

[15] Y. Shen, C. C. Yin, and K. C. Yuen, "Alternative approach to the optimality of the threshold strategy for spectrally negative Lévy processes," Acta Mathematicae Applicatae Sinica, English Series, vol. 29, no. 4, pp. 705-716, 2013.

[16] W. G. Yu, "Randomized dividends in a discrete insurance risk model with stochastic premium income," Mathematical Problems in Engineering, vol. 2013, Article ID 579534, 9 pages, 2013.

[17] W. G. Yu, "On the expected discounted penalty function for a Markov regime-switching insurance risk model with stochastic premium income," Discrete Dynamics in Nature and Society, vol. 2013, Article ID 320146, 9 pages, 2013.

[18] W. G. Yu, "Some results on absolute ruin in the perturbed insurance risk model with investment and debit interests," Economic Modelling, vol. 31, pp. 625-634, 2013.

[19] C. C. Yin and K. C. Yuen, "Exact joint laws associated with spectrally negative Lévy processes and applications to insurance risk theory," Frontiers of Mathematics in China, vol. 9, no. 6, pp. 1453-1471, 2014.

[20] C. C. Yin and K. C. Yuen, "Optimality of the threshold dividend strategy for the compound Poisson model," Statistics \& Probability Letters, vol. 81, no. 12, pp. 1841-1846, 2011.

[21] Y. X. Zhao and D. J. Yao, "Optimal dividend and capital injection problem with a random time horizon and a ruin penalty in the dual model," Applied Mathematics-A Journal of Chinese Universities Series B, vol. 30, no. 3, pp. 325-339, 2015.

[22] X. X. Zheng, J. M. Zhou, and Z. Y. Sun, "Robust optimal portfolio and proportional reinsurance for an insurer under a CEV model," Insurance: Mathematics and Economics, vol. 67, pp. 77-87, 2016.

[23] Y. Huang, X. Q. Yang, and J. M. Zhou, “Optimal investment and proportional reinsurance for a jump-diffusion risk model with constrained control variables," Journal of Computational and Applied Mathematics, vol. 296, pp. 443-461, 2016.

[24] Y. Q. Li, C. C. Yin, and X. W. Zhou, "On the last exit times for spectrally negative Lévy processes," Journal of Applied Probability, vol. 54, no. 2, pp. 474-489, 2017.

[25] Z. M. Zhang, E. C. Cheung, and H. L. Yang, "Lévy insurance risk process with Poissonian taxation," Scandinavian Actuarial Journal, vol. 2017, no. 1, pp. 51-87, 2017.

[26] W. G. Yu, Y. J. Huang, and C. R. Cui, "The absolute ruin insurance risk model with a threshold dividend strategy," Symmetry, vol. 10, no. 9, pp. 1-19, 2018. 
[27] Y. Zeng, D. P. Li, Z. Chen, and Z. Yang, "Ambiguity aversion and optimal derivative-based pension investment with stochastic income and volatility," Journal of Economic Dynamics and Control, vol. 88, pp. 70-103, 2018.

[28] Y. Zeng, D. P. Li, and A. L. Gu, "Robust equilibrium reinsuranceinvestment strategy for a mean-variance insurer in a model with jumps," Insurance: Mathematics and Economics, vol. 66, pp. 138152, 2016.

[29] D. P. Li, Y. Zeng, and H. L. Yang, "Robust optimal excess-of-loss reinsurance and investment strategy for an insurer in a model with jumps," Scandinavian Actuarial Journal, vol. 2018, no. 2, pp. 145-171, 2018.

[30] H. Dong, C. C. Yin, and H. S. Dai, "Spectrally negative Lévy risk model under Erlangized barrier strategy," Journal of Computational and Applied Mathematics, vol. 351, pp. 101-116, 2019.

[31] R. Mnatsakanov, L. L. Ruymgaart, and F. H. Ruymgaart, "Nonparametric estimation of ruin probabilities given a random sample of claims," Mathematical Methods of Statistics, vol. 17, no. 1, pp. 35-43, 2008.

[32] E. Masiello, "On semiparametric estimation of ruin probabilities in the classical risk model," Scandinavian Actuarial Journal, vol. 2014, no. 4, pp. 283-308, 2014.

[33] Z. M. Zhang, H. L. Yang, and H. Yang, "On a nonparametric estimator for ruin probability in the classical risk model," Scandinavian Actuarial Journal, vol. 2014, no. 4, pp. 309-338, 2014.

[34] Z. M. Zhang, "Nonparametric estimation of the finite time ruin probability in the classical risk model," Scandinavian Actuarial Journal, vol. 2017, no. 5, pp. 452-469, 2017.

[35] Y. Yang, W. Su, and Z. M. Zhang, "Estimating the discounted density of the deficit at ruin by Fourier cosine series expansion," Statistics \& Probability Letters, vol. 146, pp. 147-155, 2019.

[36] Y. Shimizu, "Non-parametric estimation of the Gerber-Shiu function for the Wiener-Poisson risk model," Scandinavian Actuarial Journal, no. 1, pp. 56-69, 2012.

[37] Z. M. Zhang, "Approximating the density of the time to ruin via Fourier-cosine series expansion," ASTIN Bulletin, vol. 47, no. 1, pp. 169-198, 2017.

[38] Z. M. Zhang, "Estimating the gerber-shiu function by FourierSinc series expansion," Scandinavian Actuarial Journal, vol. 2017, no. 10, pp. 898-919, 2017.

[39] Z. M. Zhang and W. Su, "A new efficient method for estimating the Gerber-Shiu function in the classical risk model," Scandinavian Actuarial Journal, vol. 2018, no. 5, pp. 426-449, 2018.

[40] Z. M. Zhang and W. Su, "Estimating the Gerber-Shiu function in a Lévy risk model by Laguerre series expansion," Journal of Computational and Applied Mathematics, vol. 346, pp. 133-149, 2019.

[41] W. Su, Y. D. Yong, and Z. M. Zhang, "Estimating the GerberShiu function in the perturbed compound Poisson model by Laguerre series expansion," Journal of Mathematical Analysis and Applications, vol. 469, no. 2, pp. 705-729, 2019.

[42] Y. Shimizu, "Estimation of the expected discounted penalty function for Lévy insurance risks," Mathematical Methods of Statistics, vol. 20, no. 2, pp. 125-149, 2011.

[43] Z. M. Zhang and H. L. Yang, "Nonparametric estimate of the ruin probability in a pure-jump Lévy risk model," Insurance: Mathematics and Economics, vol. 53, no. 1, pp. 24-35, 2013.

[44] Z. M. Zhang and H. L. Yang, "Nonparametric estimation for the ruin probability in a Lévy risk model under low-frequency observation," Insurance: Mathematics and Economics, vol. 59, pp. 168-177, 2014.

[45] Y. Shimizu and Z. M. Zhang, "Estimating Gerber-Shiu functions from discretely observed Lévy driven surplus," Insurance: Mathematics and Economics, vol. 74, pp. 84-98, 2017.

[46] Y. F. Wang and C. C. Yin, "Approximation for the ruin probabilities in a discrete time risk model with dependent risks," Statistics \& Probability Letters, vol. 80, no. 17-18, pp. 1335-1342, 2010.

[47] K. C. Yuen and C. C. Yin, "Asymptotic results for tail probabilities of sums of dependent and heavy-tailed random variables," Chinese Annals of Mathematics, Series B, vol. 33, no. 4, pp. 557568, 2012.

[48] H. Dong and C. C. Yin, "Complete monotonicity of the probability of ruin and de Finetti's dividend problem," Journal of Systems Science \& Complexity, vol. 25, no. 1, pp. 178-185, 2012.

[49] C. C. Yin, Y. Shen, and Y. Z. Wen, "Exit problems for jump processes with applications to dividend problems," Journal of Computational and Applied Mathematics, vol. 245, pp. 30-52, 2013.

[50] Y. F. Wang, C. C. Yin, and X. S. Zhang, "Uniform estimate for the tail probabilities of randomly weighted sums," Acta Mathematicae Applicatae Sinica, English Series, vol. 30, no. 4, pp. 1063-1072, 2014.

[51] Y. X. Zhao, R. M. Wang, and D. J. Yao, "Optimal dividend and equity issuance in the perturbed dual model under a penalty for ruin," Communications in Statistics-Theory and Methods, vol. 45, no. 2, pp. 365-384, 2016.

[52] P. Pavone, G. Spampinato, V. Tomaselli et al., "Map of the habitats of the EEC directive 92/43 in the biotopes of the syracuse province (eastern sicily)," Fitosociologia, vol. 44, no. 2, supplement 1, pp. 183-193, 2007.

[53] M. Zhou, K. C. Yuen, and C. C. Yin, "Optimal investment and premium control in a nonlinear diffusion model," Acta Mathematicae Applicatae Sinica, English Series, vol. 33, no. 4, pp. 945-958, 2017.

[54] R. Costa and P. Pavone, "Diachronic biodiversity analysis of a metropolitan area in the Mediterranean region," Acta Horticulturae, no. 1215, pp. 49-52, 2018.

[55] R. Costa and P. Pavone, "Invasive plants and natural habitats: the role of alien species in the urban vegetation," Acta Horticulturae, no. 1215, pp. 57-60, 2018.

[56] C. C. Yin, "Remarks on equality of two distributions under some partial orders," Acta Mathematicae Applicatae Sinica, English Series, vol. 34, no. 2, pp. 274-280, 2018.

[57] R. M. S. Costa, T. van Andel, P. Pavone, and S. Pulvirenti, "The pre-Linnaean herbarium of Paolo Boccone (1633-1704) kept in Leiden (the Netherlands) and its connections with the imprinted one in Paris," Plant Biosystems, vol. 152, no. 3, pp. 489-500, 2018.

[58] S. Pulvirenti, P. Pavone, R. A. Carbonaro, and R. M. Costa, “ Taxonomic study of the plants to be found in the only ", Plant Biosystems - An International Journal Dealing with all Aspects of Plant Biology, vol. 151, no. 4, pp. 745-759, 2016.

[59] S. Pulvirenti, P. Pavone, R. A. Carbonaro, and R. M. Costa, "The controversial biography of Paolo Boccone (1633-1704) and his "Grand Tour" from the Mediterranean to northern Europe," Plant Biosystems - An International Journal Dealing with all Aspects of Plant Biology, vol. 151, no. 3, pp. 377-380, 2017.

[60] P. G. Lemarié-Rieusset and Y. Meyer, "Ondelettes et bases hilbertiennes," Revista Matemática Iberoamericana, vol. 2, no. 1, pp. 1-18, 1986. 
[61] I. Daubechies, Ten Lectures on Wavelets, SIAM, Philadelphia, Pa, USA, 1992.

[62] M. Abramowitz and I. A. Stegun, Handbook of Mathematical Functions with Formulas, Graphs, and Mathematical Tables, National Bureau of Standards Applied Mathematics Series, U.S. Government Printing Office, Washington DC, Wash, USA, 1964.

[63] F. Comte and V. Genon-Catalot, "Nonparametric estimation for pure jump Lévy processes based on high frequency data," Stochastic Processes and Their Applications, vol. 119, no. 12, pp. 4088-4123, 2009.

[64] B. Bongioanni and J. L. Torrea, "What is a Sobolev space for the Laguerre function systems?” Studia Mathematica, vol. 192, no. 2, pp. 147-172, 2009.

[65] F. Comte, C.-A. Cuenod, M. Pensky, and Y. Rozenholc, "Laplace deconvolution on the basis of time domain data and its application to dynamic contrast-enhanced imaging," Journal of the Royal Statistical Society: Series B (Statistical Methodology), vol. 79, no. 1, pp. 69-94, 2017. 


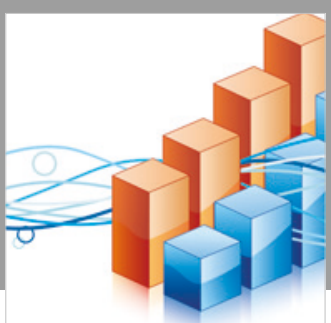

Advances in

Operations Research

\section{-n-m}
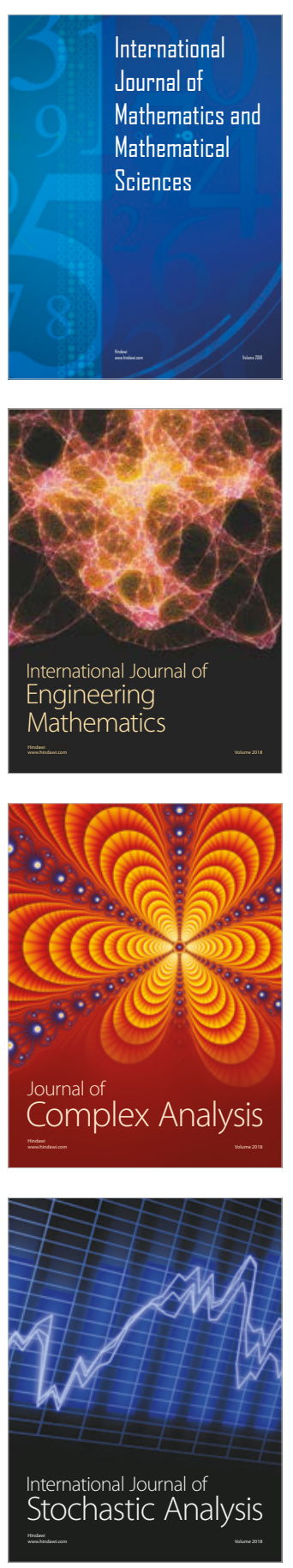
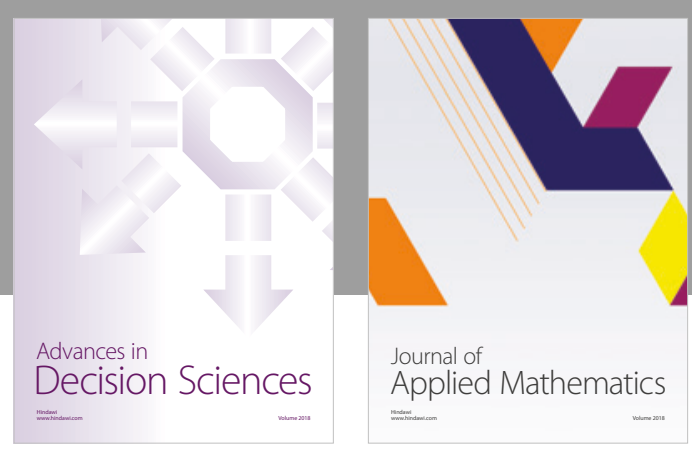

Journal of

Applied Mathematics
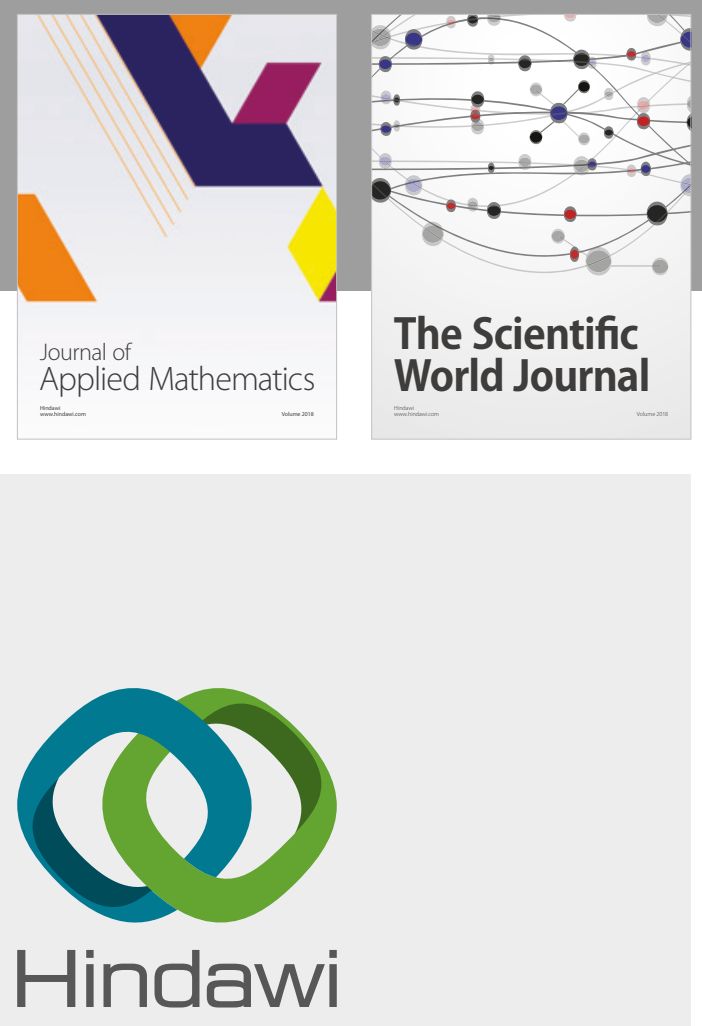

Submit your manuscripts at

www.hindawi.com

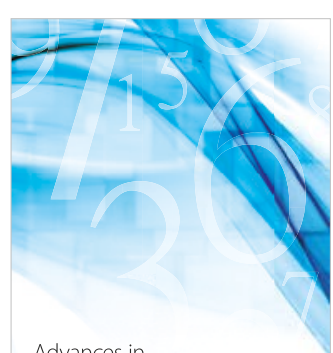

Advances in
Numerical Analysis
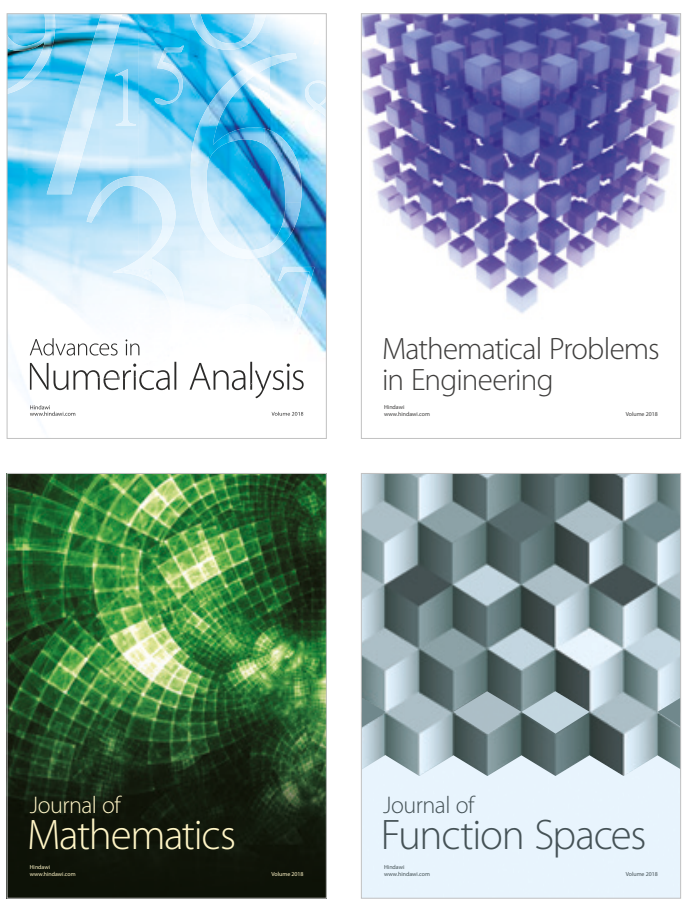

Mathematical Problems in Engineering

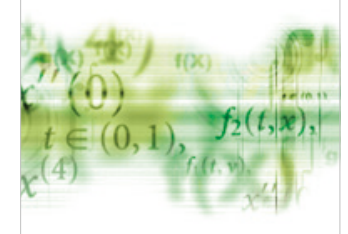

International Journal of

Differential Equations

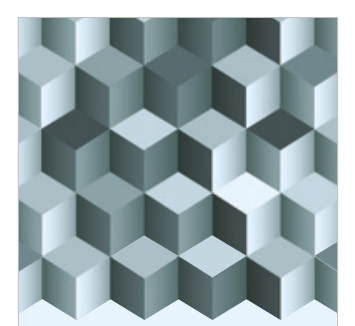

Journal of

Function Spaces

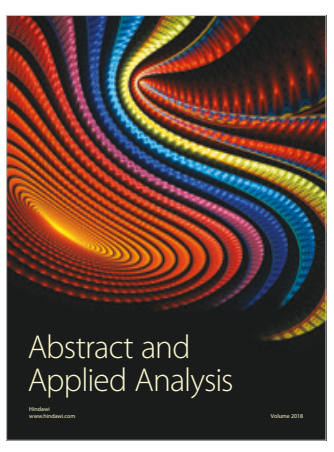

The Scientific

World Journal

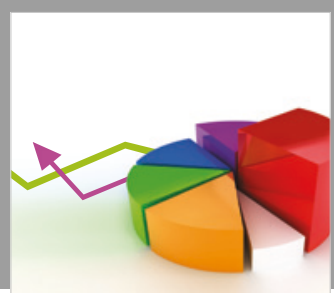

Journal of

Probability and Statistics
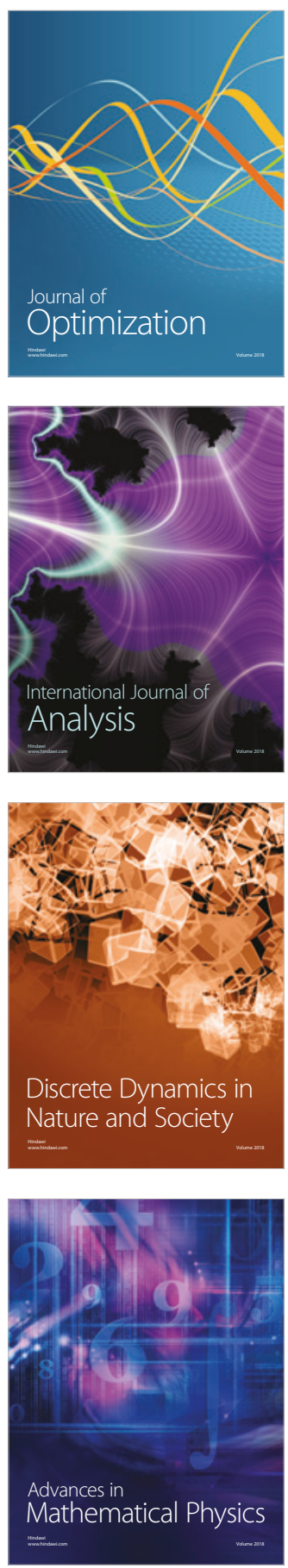